\title{
Graph Controllability Classes for the Laplacian Leader-Follower Dynamics
}

\author{
Cesar O. Aguilar and Bahman Gharesifard
}

\begin{abstract}
In this paper, we consider the problem of obtaining graph-theoretic characterizations of controllability for the Laplacian-based leader-follower dynamics. Our developments rely on the notion of graph controllability classes, namely, the classes of essentially controllable, completely uncontrollable, and conditionally controllable graphs. In addition to the topology of the underlying graph, the controllability classes rely on the specification of the control vectors; our particular focus is on the set of binary control vectors. The choice of binary control vectors is naturally adapted to the Laplacian dynamics, as it captures the case when the controller is unable to distinguish between the followers and, moreover, controllability properties are invariant under binary complements. We prove that the class of essentially controllable graphs is a strict subset of the class of asymmetric graphs and provide numerical results that suggests that the ratio of essentially controllable graphs to asymmetric graphs increases as the number of vertices increases. Although graph symmetries play an important role in graph-theoretic characterizations of controllability, we provide an explicit class of asymmetric graphs that are completely uncontrollable, namely the class of block graphs of Steiner triple systems. We prove that for graphs on 4 and 5 vertices, a repeated Laplacian eigenvalue is a necessary condition for complete uncontrollability but, however, show through explicit examples that for 8 and 9 vertices, a repeated eigenvalue is not necessary for complete uncontrollability. For the case of conditional controllability, we give an easily checkable necessary condition that identifies a class of homogeneous binary control vectors that result in a two-dimensional controllable subspace. Finally, we give a lowerbound on the rank of the controllability matrix for the Laplacian dynamics. Several constructive examples demonstrate our results.
\end{abstract}

\section{INTRODUCTION}

Many modern engineering and economic systems consist of collections of smaller subsystems interconnected to each other over a communication network. Examples include distributed energy resources, oscillator synchronization, distributed robotic networks [2], [3], [4], and also cascades of information and opinions in social networks [5]. Due to the large number of applications, in recent years there has been a surge of activity within the control theory community to understand how the network structure of multi-agent systems affects the fundamental properties of controllability and stabilizability. With regards to controllability, a graph theoretic characterization of linear networked control systems has been fully developed in [6] via the notion of structural controllability. In spite of this characterization, the recent attention within the control community on the so-called Laplacian dynamics has

Cesar O. Aguilar is with the Department of Mathematics, California State University, Bakersfield, CA 93311, USA caguilar24@csub. edu and Bahman Gharesifard is with the Department of Mathematics and Statistics, Queen's University, Kingston, ON K7L 3N6, Canada, bahman@mast. queensu.ca. An incomplete version of this paper is submitted for presentation at the IEEE Control and Decision Conference 2014 as [1]. resulted in an extensive literature on controllability properties for this class of control systems, see of example [7], [8], [9], [10], [11], [12]. Specifically, starting with a Laplacian-based dynamics, a subset of the agents are classified as leaders and act as control agents that can change the dynamics of the network. The remaining agents, called the followers, are indirectly controlled by the leaders via the connectivity of the network. Most of the effort has gone to characterizing graph-theoretic conditions under which such systems are uncontrollable.

\section{A. Literature review}

The controllability of leader-follower network dynamics was first considered in [7], where a characterization of controllability using spectral analysis of the system matrix was given. Using a graph-theoretic approach, in [8] it was shown that for a single leader agent, symmetries present in the network that preserve the leader's neighbors results in uncontrollability. Moreover, in the case of multiple leaders, a necessary condition for controllability was given using equitable graph partitions. In [9], it is shown that connectivity of the network is necessary for controllability and two uncontrollable network topologies are characterized. In [10], various sufficient and necessary conditions for controllability are given for a network tree topology. In [11], sufficient and necessary conditions for controllability (and observability) of multi-input Laplacian dynamics for path and cycle network topologies are given in terms of modular arithmetic relations. In [12], a comprehensive study was undertaken of the controllability (and observability) properties of grid graphs. In particular, necessary and sufficient conditions are given that characterize the set of nodes that result in controllability.

The controllability problem for graphs has received interest outside the control community. In [13], [14], the adjacency matrix is used instead of the Laplacian matrix to study the graph controllability problem. Explicitly, a "controllable graph" in [13], [14] is a graph whose adjacency matrix has distinct eigenvalues and no eigenvector of the adjacency matrix is orthogonal to the all ones vector. Hence, the work in [13], [14] investigates controllability when all of the nodes are chosen as leaders and the system matrix is given by the adjacency matrix. A similar approach is taken in [15], but now one is allowed to control possibly only a subset of the nodes. We note that when the system matrix is the Laplacian instead of the adjacency matrix, all graphs of order $n \geq 2$ are uncontrollable according to the definition of "controllable graphs" given in [13], [14], [15]. This follows from the well-known fact that the all ones vector is an eigenvector of the Laplacian matrix, and therefore orthogonal to the other eigenvectors.

The line of research in [7], [8], [9], [10] takes the point of view that the states of the leaders act as inputs to the 
follower agents and the dynamics of the leaders are ignored. As a result, the controllability analysis is undertaken on a reduced order system. On the other hand, the line of research in [11], [12], [16], [17] takes the point of view that the leaders continue to follow the Laplacian-based dynamics and the external controls on the leaders influence the entire network through the interaction of the leaders with the followers. It is, however, an easy exercise to show that the former approach is a special case of the latter (see Remark 3.1). In this paper, our approach is more closely aligned with [11], [12], [16], [17].

\section{B. Statement of contributions}

The contributions of this paper are the following. To better understand the role of topological graph obstructions to controllability for Laplacian-based leader-follower systems, we introduce graph controllability classes, namely, essentially controllable graphs, completely uncontrollable graphs, and conditionally controllable graphs. These definitions rely on the specification of the control vectors and we focus primarily on the case of binary control vectors. We show that with this choice of control vectors, controllability is invariant under binary complements for Laplacian-based leader-follower dynamics.

As our first result on graph controllability classes, we prove that none of the essentially controllable graphs contain a nonidentity graph automorphism, i.e., all such graphs are asymmetric. As a by-product, the so-called minimal controllability problems [18] are solvable for this class. We also provide numerical results that suggest that the ratio of essentially controllable graphs to asymmetric graphs tends to one as the number of vertices increases.

We then provide an explicit class of graphs, namely the block graphs of Steiner triple systems, that are asymmetric yet completely uncontrollable, and thus showing that essentially controllable graphs form a strict subset of asymmetric graphs. Although symmetry plays an important role in graph-theoretic characterizations of controllability [8], this result and the fact that asymmetry is typical in finite graphs [19], suggests that the current focus in the literature on characterizing graph uncontrollability by identifying graph symmetries targets a narrow non-generic scenario. We prove that for connected graphs with four or five vertices, a repeated eigenvalue is a necessary condition for complete uncontrollability but show through explicit examples that for $n \geq 8$ this condition is in general not necessary. As a by-product of our results, we give a sufficient condition for complete uncontrollability in terms of the eigenvectors of the Laplacian matrix and construct a class of non-regular completely uncontrollable graphs.

We then provide a sufficient condition for conditional controllability. Specifically, we identify a class of binary vectors that we call homogeneous that result in a two-dimensional controllable subspace. As an example, we show that the 3regular asymmetric Frucht graph on twelve vertices possess these homogeneous binary control vectors. We then provide a lower-bound on the rank of the controllability matrix for the Laplacian based leader-follower system and discuss its implications in the controllability of path graphs. Finally, we end the paper with numerical results enumerating the distinct controllability classes for graphs from order $n=2$ to $n=9$. Throughout the paper, several examples demonstrate the results.

\section{Organization}

The remainder of this paper is organized as follows. In Section II we establish some notation and present necessary definitions from graph theory along with a result on the linear controllability for diagonalizable system matrices. In Section III, after establishing some preliminary results, we discuss the motivation of this paper as it relates to the existing literature on graph symmetries and uncontrollability of networked systems, and then introduce our graph controllability classes. Section IV contain our main results. Finally, in Section $\mathrm{V}$, we make concluding remarks and discuss ideas for future work.

\section{PRELiminaries}

The set of natural numbers is denoted by $\mathbb{N}$ and we set $\mathbb{N}_{0}=\{0\} \cup \mathbb{N}$. Matrices will be denoted using upper case bold letters such as $\mathbf{A}, \mathbf{F}, \mathbf{L}$, and vectors using lower case bold letters such as $\mathbf{x}, \mathbf{b}, \mathbf{u}$. The transpose of $\mathbf{A}$ is denoted by $\mathbf{A}^{T}$. The cardinality of a finite set $S$ is denoted by $|S|$. If $S \subset R$ then the complement of $S$ in $R$ is denoted by $R \backslash S$. The standard basis vectors in $\mathbb{R}^{n}$ are denoted by $\mathbf{e}_{1}, \mathbf{e}_{2}, \ldots, \mathbf{e}_{n}$. Finally, given $\mathbf{u}, \mathbf{v} \in \mathbb{R}^{n}$, we write that $\mathbf{u} \perp \mathbf{v}$ if $\mathbf{u}$ and $\mathbf{v}$ are orthogonal in the standard inner product of $\mathbb{R}^{n}$, i.e., $\mathbf{v}^{T} \mathbf{u}=\mathbf{u}^{T} \mathbf{v}=0$. More generally, if $W \subset \mathbb{R}^{n}$, we write $\mathbf{u} \perp W$ if $\mathbf{u} \perp \mathbf{w}$ for each $\mathbf{w} \in W$.

\section{A. Graph theory}

Our notation from graph theory is standard and follows the notation in [20], [21]. By a graph we mean a pair $\mathcal{G}=(\mathcal{V}, \mathcal{E})$ consisting of a finite vertex set $\mathcal{V}$ and an edge set $\mathcal{E} \subseteq[\mathcal{V}]^{2}:=$ $\{\{v, w\} \mid v, w \in \mathcal{V}\}$. We consider only simple graphs, i.e., unweighted, undirected, with no loops or multiple edges. The order of the graph $\mathcal{G}$ is the cardinality of its vertex set $\mathcal{V}$. The neighbors of $v \in \mathcal{V}$ is the set $\mathcal{N}_{v}:=\{w \in \mathcal{V} \mid\{v, w\} \in \mathcal{E}\}$ and the degree of $v$ is $d_{v}:=\left|\mathcal{N}_{v}\right|$. A path in $\mathcal{G}$ of length $k$ is a subgraph of $\mathcal{G}$ consisting of vertices $\left\{v_{0}, v_{1}, \ldots, v_{k}\right\} \subset \mathcal{V}$ and edges $\left\{\left\{v_{0}, v_{1}\right\},\left\{v_{1}, v_{2}\right\}, \ldots,\left\{v_{k-1}, v_{k}\right\}\right\} \subset \mathcal{E}$, where all the $v_{i}$ are distinct. For such a path, $v_{0}$ and $v_{k}$ are called the terminal vertices. Given vertices $u, v \in \mathcal{V}$, we define the distance $\mathrm{d}_{\mathcal{G}}(u, v)$ between $u$ and $v$ as the length of a shortest path whose terminal vertices are $u$ and $v$. A graph $\mathcal{G}$ is connected if there is a path between any pair of vertices.

Henceforth, when not explicitly stated, we fix an ordering on the vertex set $\mathcal{V}$ and thus, without loss of generality, we take $\mathcal{V}=\{1, \ldots, n\}$, where $n$ is the order of $\mathcal{G}$. The adjacency matrix of $\mathcal{G}$ is the $n \times n$ matrix $\mathbf{A}$ defined as $\mathbf{A}_{i j}=1$ if $\{i, j\} \in \mathcal{E}$ and $\mathbf{A}_{i j}=0$ otherwise, where $\mathbf{A}_{i j}$ denotes the entry of $\mathbf{A}$ in the $i$ th row and $j$ th column. We note that if $r=\mathrm{d}_{\mathcal{G}}(i, j)$, with $i \neq j$, then $\left(\mathbf{A}^{k}\right)_{i j}=0$ for all $0 \leq k<r$ and $\left(\mathbf{A}^{r}\right)_{i j} \neq 0$.

We denote by $\mathbf{D}$ the degree matrix of $\mathcal{G}$, i.e., the diagonal matrix whose $i$ th diagonal entry is $d_{i}$. The Laplacian matrix of $\mathcal{G}$ is given by

$$
\mathbf{L}=\mathbf{D}-\mathbf{A} .
$$


The Laplacian matrix $\mathbf{L}$ is symmetric and positive semidefinite, and thus the eigenvalues of $\mathbf{L}$ can be ordered $\lambda_{1} \leq$ $\lambda_{2} \leq \cdots \leq \lambda_{n}$. The ones vector $\mathbf{1}_{n}:=\left[\begin{array}{llll}1 & 1 & \cdots & 1\end{array}\right]^{T}$ is an eigenvector of $\mathbf{L}$ with eigenvalue $\lambda_{1}=0$, and if $\mathcal{G}$ is connected then $\lambda_{1}=0$ is a simple eigenvalue of $\mathbf{L}$. We assume throughout that $\mathcal{G}$ is connected so that $0<\lambda_{2}$. For our purposes, by the eigenvalues (eigenvectors) of a graph $\mathcal{G}$ we mean the eigenvalues (eigenvectors) of its Laplacian matrix L.

A mapping $\varphi: \mathcal{V} \rightarrow \mathcal{V}$ is an automorphism of $\mathcal{G}$ if it is a bijection and $\{i, j\} \in \mathcal{E}$ implies that $\{\varphi(i), \varphi(j)\} \in \mathcal{E}$. The order of an automorphism $\varphi$ is the smallest positive integer $k$ such that the $k$-fold composition of $\varphi$ with itself is the identity automorphism. An automorphism $\varphi$ of $\mathcal{G}$ induces a linear transformation on $\mathbb{R}^{n}$, denoted by $\mathbf{P}_{\varphi}$ or just $\mathbf{P}$ when $\varphi$ is understood, whose matrix representation in the standard basis is a permutation matrix, i.e., as a linear mapping $\varphi$ acts as a permutation on the standard basis $\left\{\mathbf{e}_{1}, \ldots, \mathbf{e}_{n}\right\}$ of $\mathbb{R}^{n}$. It is well-known that $\varphi$ is an automorphism of $\mathcal{G}$ if and only if $\mathbf{P A}=\mathbf{A P}$. Moreover, an automorphism $\mathbf{P}$ preserves degree of vertices, and therefore $d_{i}=d_{\varphi(i)}$ for every $i \in\{1,2, \ldots, n\}$, i.e., $\mathbf{P D}=\mathbf{D P}$. It follows that an automorphism $\mathbf{P}$ of $\mathcal{G}$ also satisfies $\mathbf{P L}=\mathbf{L P}$.

A graph is called $k$-regular if all its vertices have degree $k \in \mathbb{N}$. A $k$-regular graph $\mathcal{G}=(\mathcal{V}, \mathcal{E})$ is called strongly regular if there exists $\lambda, \mu \in \mathbb{N}$ such that

i) $\left|\mathcal{N}_{v} \cap \mathcal{N}_{u}\right|=\lambda$, for every $v \in \mathcal{V}$ and every $u \in \mathcal{N}_{v}$, and

ii) $\left|\mathcal{N}_{v} \cap \mathcal{N}_{u}\right|=\mu$, for every $v \in \mathcal{V}$ and every $u \notin \mathcal{N}_{v}$.

It is known that strongly regular graphs have exactly three Laplacian eigenvalues [22]. A strongly regular graph will be denoted by $\operatorname{SRG}(n, k, \lambda, \mu)$.

\section{B. Diagonalizability and linear controllability}

Given a matrix $\mathbf{F} \in \mathbb{R}^{n \times n}$ and vector $\mathbf{b} \in \mathbb{R}^{n}$, we denote by $\langle\mathbf{F} ; \mathbf{b}\rangle$ the smallest $\mathbf{F}$-invariant subspace containing b. It is well-known that $\langle\mathbf{F} ; \mathbf{b}\rangle=\operatorname{span}\left\{\mathbf{F}^{k} \mathbf{b} \mid k \in \mathbb{N}_{0}\right\}$, and that if $\operatorname{dim}\langle\mathbf{F} ; \mathbf{b}\rangle=k+1$ then $\left\{\mathbf{b}, \mathbf{F b}, \ldots, \mathbf{F}^{k} \mathbf{b}\right\}$ is a basis for $\langle\mathbf{F} ; \mathbf{b}\rangle$. The pair $(\mathbf{F}, \mathbf{b})$ is called controllable if $\operatorname{dim}\langle\mathbf{F} ; \mathbf{b}\rangle=n$. The following result characterizes the controllability of single-input linear systems $(\mathbf{F}, \mathbf{b})$ when $\mathbf{F}$ is diagonalizable.

Proposition 2.1: (Controllability and eigenvalue multiplicity): Let $\mathbf{F} \in \mathbb{R}^{n \times n}$ be diagonalizable.

(i) For any open set $\mathrm{B} \subset \mathbb{R}^{n}$, the pair $(\mathbf{F}, \mathbf{b})$ is uncontrollable for every $\mathbf{b} \in \mathrm{B}$ if and only if $\mathbf{F}$ has a repeated eigenvalue.

(ii) Suppose that $\mathbf{F}$ has distinct eigenvalues and let $\mathbf{U}$ be a matrix whose columns are linearly independent eigenvectors of $\mathbf{F}$. If $\mathbf{b} \in \mathbb{R}^{n}$ then the dimension of $\langle\mathbf{F} ; \mathbf{b}\rangle$ is equal to the number of nonzero components of $\mathbf{v}=\mathbf{U}^{-1} \mathbf{b}$. In particular, $(\mathbf{F}, \mathbf{b})$ is controllable if and only if no component of $\mathbf{v}$ is zero.

The proof of (i) follows from the properties of the determinant and for the proof of (ii) see for instance [7].

\section{Problem Statement AND GRAPH Controllability Classes}

Let $\mathcal{G}=(\mathcal{V}, \mathcal{E})$ be a graph with vertex set $\mathcal{V}=$ $\{1,2, \ldots, n\}$. The Laplacian dynamics on $\mathcal{G}$ is the linear system

$$
\dot{\mathbf{x}}(t)=-\mathbf{L x}(t),
$$

where $\mathbf{x} \in \mathbb{R}^{n}, t \in \mathbb{R}$, and $\mathbf{L}$ is the Laplacian matrix of $\mathcal{G}$. Suppose that a nonempty subset of the vertices $\widetilde{\mathcal{V}} \subset \mathcal{V}$ are actuated by a single control $u:[0, \infty) \rightarrow \mathbb{R}$ and consider the resulting single-input linear control system. Explicitly, let $\mathbf{b}=\left[\begin{array}{llll}b_{1} & b_{2} & \cdots & b_{n}\end{array}\right]^{T} \in\{0,1\}^{n}$ be the binary vector such that $\widetilde{\mathcal{V}}=\mathcal{V}_{\mathbf{b}}:=\left\{i \in \mathcal{V} \mid b_{i}=1\right\}$, and consider the single-input linear control system

$$
\dot{\mathbf{x}}(t)=-\mathbf{L x}(t)+\mathbf{b} u(t) .
$$

The vertices $\mathcal{V}_{\mathbf{b}}$ are seen as control or leader nodes and influence the remaining follower nodes $\mathcal{V} \backslash \mathcal{V}_{\mathbf{b}}$ through the control signal $u(\cdot)$ and the connectivity of the network. A motivation for the set of binary control vectors is that it captures the scenario of when an external agent connected to the nodes $\mathcal{V}_{\mathbf{b}}$ is unable to distinguish between its followers. Hence, all the followers receive the same control input from the leader. The reason for choosing the Laplacian dynamics (1) is that it serves as a benchmark problem for studying distributed control systems. The problem is also of independent theoretical interest because it reveals useful information about the set of eigenvectors of the Laplacian matrix of a graph [15].

From a controls design perspective, it would of course be desirable to select the leader nodes so that the pair $(\mathbf{L}, \mathbf{b})$ is controllable. First, note that choosing $\mathbf{b}=\mathbf{1}_{n}$ results in a controllable pair $(\mathbf{L}, \mathbf{b})$ if and only if $n=1$ since $\mathbf{L} \mathbf{1}_{n}=\mathbf{0}_{n}$. More generally, a direct application of Proposition 2.1(ii) for the Laplacian dynamics yields the following result.

Corollary 3.1 ([7]): (Necessary and sufficient condition for controllability of Laplacian dynamics): Consider the controlled Laplacian dynamics (1) with $\mathbf{b} \in \mathbb{R}^{n}$ and assume that $\mathbf{L}$ has no repeated eigenvalues. Then the pair $(\mathbf{L}, \mathbf{b})$ is controllable if and only if $\mathbf{b}$ is not orthogonal to any eigenvector of $\mathbf{L}$.

Although Corollary 3.1 provides a general necessary and sufficient condition for controllability in terms of the graph Laplacian eigenvectors, the problem that we consider is in obtaining controllability conditions in terms of the topological structure of the graph. Graph-theoretic characterizations of controllability for leader-follower multi-agent systems was first considered in [8] in terms of the automorphism group of a graph. Following [8], we say that $\mathbf{b} \in\{0,1\}^{n}$ is leader symmetric if there exists a non-trivial automorphism $\varphi: \mathcal{V} \rightarrow \mathcal{V}$ of $\mathcal{G}$ that leaves the leader nodes $\mathcal{V}_{\mathbf{b}}$ invariant, i.e., $\mathbf{P}_{\varphi}(\mathbf{b})=\mathbf{b}$. It is straightforward to verity that the definition of leader symmetry given in [8] is equivalent to the one given here. The following result of [8] links leader symmetry and uncontrollability (a short alternative proof is given in the Appendix).

Proposition 3.1: (Leader symmetry and uncontrollability): Consider the controlled Laplacian dynamics (1) with $\mathbf{b} \in$ $\{0,1\}^{n}$. If $\mathbf{b}$ is leader symmetric then $(\mathbf{L}, \mathbf{b})$ is uncontrollable.

As shown in [8, Proposition 5.9], leader symmetry is not a necessary condition for uncontrollability. Figure 1(a) displays the graph on $n=6$ vertices that is used in [8] to show this fact. Unfortunately, this example is not illuminating in the quest for obtaining graph-theoretic characterizations of controllability because the leader nodes are chosen so that $\mathbf{b}=\mathbf{1}_{n}$, i.e., 


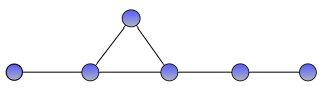

(a)

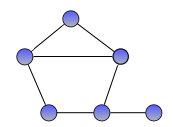

(b)

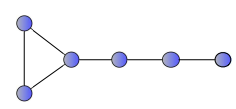

(c)
Fig. 1. (a) The example of [8], (b) an asymmetric graph on $n=6$ vertices having 14 binary vectors $\mathbf{b}$ resulting in uncontrollable Laplacian dynamics, and (c) a graph for which any binary vector $\mathbf{b}$ results in uncontrollability.

every node is actuated. As remarked above, unless $n=1$, this choice results in uncontrollability regardless of the graph topology. Interestingly, the only control vectors $\mathbf{b}$ resulting in uncontrollability for the graph in Figure 1(a) are the trivial ones, i.e. $\mathbf{b}=\mathbf{0}_{n}$ or $\mathbf{b}=\mathbf{1}_{n}$. In view of the fact that asymmetry is typical in finite graphs [19], it is natural then to ask what graph-theoretic obstructions to controllability exist other than symmetry. For example, consider the asymmetric graph on $n=6$ vertices displayed in Figure 1(b). Of the $2^{n}-2=62$ non-trivial choices of $\mathbf{b}$, there are 14 that result in uncontrollability, namely:

$$
\begin{aligned}
& \mathbf{b}_{1}=\left[\begin{array}{llllll}
1 & 1 & 1 & 0 & 0 & 0
\end{array}\right]^{T}, \quad \mathbf{b}_{2}=\left[\begin{array}{llllll}
0 & 0 & 0 & 1 & 1 & 1
\end{array}\right]^{T}, \\
& \mathbf{b}_{3}=\left[\begin{array}{llllll}
1 & 1 & 0 & 1 & 0 & 0
\end{array}\right]^{T}, \quad \mathbf{b}_{4}=\left[\begin{array}{llllll}
0 & 0 & 1 & 0 & 1 & 1
\end{array}\right]^{T}, \\
& \mathbf{b}_{5}=\left[\begin{array}{llllll}
0 & 1 & 1 & 1 & 0 & 0
\end{array}\right]^{T}, \quad \mathbf{b}_{6}=\left[\begin{array}{llllll}
1 & 0 & 0 & 0 & 1 & 1
\end{array}\right]^{T}, \\
& \mathbf{b}_{7}=\left[\begin{array}{llllll}
0 & 1 & 0 & 0 & 1 & 0
\end{array}\right]^{T}, \quad \mathbf{b}_{8}=\left[\begin{array}{llllll}
1 & 0 & 1 & 1 & 0 & 1
\end{array}\right]^{T}, \\
& \mathbf{b}_{9}=\left[\begin{array}{llllll}
1 & 0 & 1 & 0 & 1 & 0
\end{array}\right]^{T}, \quad \mathbf{b}_{10}=\left[\begin{array}{llllll}
0 & 1 & 0 & 1 & 0 & 1
\end{array}\right]^{T}, \\
& \mathbf{b}_{11}=\left[\begin{array}{llllll}
1 & 0 & 0 & 1 & 1 & 0
\end{array}\right]^{T}, \quad \mathbf{b}_{12}=\left[\begin{array}{llllll}
0 & 1 & 1 & 0 & 0 & 1
\end{array}\right]^{T}, \\
& \mathbf{b}_{13}=\left[\begin{array}{llllll}
0 & 0 & 1 & 1 & 1 & 0
\end{array}\right]^{T}, \quad \mathbf{b}_{14}=\left[\begin{array}{llllll}
1 & 1 & 0 & 0 & 0 & 1
\end{array}\right]^{T} \text {. }
\end{aligned}
$$

The control vectors $\mathbf{b}_{7}$ and $\mathbf{b}_{8}$ result in a two-dimensional controllable subspace, while the other control vectors all result in a five-dimensional controllable subspace. On the other hand, for the graph on $n=6$ vertices displayed in Figure 1(c), any choice of $\mathbf{b} \in\{0,1\}^{n}$ results in uncontrollability. Clearly, the graph displayed in Figure 1(c) has a non-trivial symmetry but symmetry plays no role in the lack of controllability for every control vector $\mathbf{b} \in\{0,1\}^{n}$. In fact, as we will show, there exist asymmetric graphs such that no matter the choice of $\mathbf{b} \in\{0,1\}^{n}$ the pair $(\mathbf{L}, \mathbf{b})$ is uncontrollable.

Our previous discussion naturally leads to the definition of the following three graph controllability classes.

Definition 3.1: (Graph controllability classes): Let $\mathcal{G}$ be a connected graph with Laplacian matrix $\mathbf{L}$ and let $\mathrm{B} \subset \mathbb{R}^{n}$ be a nonempty set. Then $\mathcal{G}$ is called

(i) essentially controllable on $\mathrm{B}$ if $(\mathbf{L}, \mathbf{b})$ is controllable for every $\mathbf{b} \in \mathbf{B} \backslash \operatorname{ker}(\mathbf{L})$;

(ii) completely uncontrollable on $\mathrm{B}$ if $(\mathbf{L}, \mathbf{b})$ is uncontrollable for every $\mathbf{b} \in \mathbf{B}$;

(iii) conditionally controllable on $\mathrm{B}$, if it is neither essentially controllable nor completely uncontrollable on B.

In this paper we are mainly concerned with controllability classes on the control set $B=\{0,1\}^{n}$. Hence, when not explicitly stated, we simply call a graph $\mathcal{G}$ essentially controllable (conditionally controllable, or completely uncontrollable) if $\mathcal{G}$ is essentially controllable (conditionally controllable, or completely controllable) on $\{0,1\}^{n}$. Hence, according to Definition 3.1, the graph in Figure 1(a) is essentially controllable, the graph in Figure1(b) is conditionally controllable, and the graph in Figure1(c) is completely uncontrollable.
Remark 3.1: Let us describe the approach taken in [8] and how it relates to ours. We note that our approach is also adopted in [11], [12]. In [8], one begins with a Laplacianbased dynamics $\dot{\mathbf{x}}=-\mathbf{L x}$, selects a leader node, say $i \in$ $\{1,2, \ldots, n\}$, and considers the reduced system of followers actuated by node $i$. Explicitly, let $\mathbf{L}_{f} \in \mathbb{R}^{(n-1) \times(n-1)}$ be the matrix obtained by deleting the $i$ th row and $i$ th column of $\mathbf{L}$, and let $\mathbf{b}_{f} \in \mathbb{R}^{n-1}$ be the column vector obtained by removing the $i$ th entry of the $i$ th column of $\mathbf{L}$. The reduced system of followers considered in [8] is $\dot{\mathbf{z}}=-\mathbf{L}_{f} \mathbf{z}-\mathbf{b}_{f} u$. The system $\left(\mathbf{L}_{f}, \mathbf{b}_{f}\right)$ is controllable if and only if $\left(\mathbf{L}, \mathbf{e}_{i}\right)$ is controllable. Indeed, the dynamic extension

$$
\begin{aligned}
\dot{\mathbf{z}} & =-\mathbf{L}_{f} \mathbf{z}-\mathbf{b}_{f} \xi, \\
\dot{\xi} & =v,
\end{aligned}
$$

is controllable if and only if $\left(\mathbf{L}_{f}, \mathbf{b}_{f}\right)$ is controllable. Letting $v=-\mathbf{b}_{f}^{T} \mathbf{z}-d_{i} \xi+u$, we see that the dynamic extension is feedback equivalent to $\left(\mathbf{L}, \mathbf{e}_{i}\right)$. Hence, in relation to the problem we consider in this paper, the approach in [8] is concerned with the controllability of $(\mathbf{L}, \mathbf{b})$ in the restricted case that $\mathbf{b} \in\left\{\mathbf{e}_{1}, \mathbf{e}_{2}, \ldots, \mathbf{e}_{n}\right\} \subset\{0,1\}^{n}$. We note that the graph in Figure 1(b) is such that $\left(\mathbf{L}, \mathbf{e}_{i}\right)$ is controllable for every $\mathbf{e}_{i} \in\left\{\mathbf{e}_{1}, \ldots, \mathbf{e}_{n}\right\}$, yet as shown in the example, fails to be controllable for some $\mathbf{b} \in\{0,1\}^{n}$.

\section{GRAPH-THEORETIC CHARACTERIZATIONS OF CONTROLLABILITY CLASSES}

Before we state our main results, we provide a useful property of controllability under binary control vectors. The astute reader may have noticed that the control vectors (2) that result in uncontrollability for the graph in Figure 1(b) come in complementary pairs. To be more precise, given $\mathbf{b} \in\{0,1\}^{n}$ we let

$$
\overline{\mathbf{b}}=\mathbf{1}_{n}-\mathbf{b}
$$

be the complement of $\mathbf{b}$. As a further piece of notation, for $\mathbf{b} \in\{0,1\}^{n}$ we let $\|\mathbf{b}\|_{1}=\sum_{i=1}^{n} b_{i}$ be the number of nonzero elements of $\mathbf{b}$. With this notation we have the following result.

Proposition 4.1: (Controllability and binary complements): Let $n \geq 2$ and consider the controlled Laplacian dynamics (1) with $\mathbf{b} \in\{0,1\}^{n}$. Then the pair $(\mathbf{L}, \mathbf{b})$ is controllable if and only if the pair $(\mathbf{L}, \overline{\mathbf{b}})$ is controllable. Specifically, if $\mathbf{b} \notin\left\{\mathbf{1}_{n}, \mathbf{0}_{n}\right\}$ then $\operatorname{dim}\langle\mathbf{L} ; \mathbf{b}\rangle=\operatorname{dim}\langle\mathbf{L} ; \overline{\mathbf{b}}\rangle$.

Proof: If $\mathbf{L}$ has repeated eigenvalues, then $(\mathbf{L}, \mathbf{c})$ is uncontrollable for every $\mathbf{c} \in \mathbb{R}^{n}$, and the claim follows trivially. Hence, assume that $\mathbf{L}$ has distinct eigenvalues. Let $\mathbf{U}$ be an orthogonal matrix consisting of unit norm eigenvectors of $\mathbf{L}$ and let the first column of $\mathbf{U}$ be the eigenvector $\mathbf{u}_{1}=\frac{1}{\sqrt{n}} \mathbf{1}_{n}$. Let $\mathbf{b} \in\{0,1\}^{n} \backslash\left\{\mathbf{1}_{n}, \mathbf{0}_{n}\right\}$, let $\mathbf{v}=\mathbf{U}^{T} \mathbf{b}=\left[v_{1} \cdots v_{n}\right]^{T}$, and let $\overline{\mathbf{v}}=\mathbf{U}^{T} \overline{\mathbf{b}}=\left[\begin{array}{lll}\bar{v}_{1} & \cdots & \bar{v}_{n}\end{array}\right]^{T}$. Then

$$
\overline{\mathbf{v}}=\frac{n}{\sqrt{n}} \mathbf{e}_{1}-\mathbf{v} .
$$

From (3) we see that $\bar{v}_{i}=-v_{i}$ for all $i=2, \ldots, n$. Now, $v_{1}=\mathbf{u}_{1}^{T} \mathbf{b}=\frac{\|\mathbf{b}\|_{1}}{\sqrt{n}}$ and thus $v_{1} \neq 0$ because $\mathbf{b} \neq \mathbf{0}_{n}$. On the other hand, $\bar{v}_{1}=\frac{n-\|\mathbf{b}\|_{1}}{\sqrt{n}}$ and thus $\bar{v}_{1} \neq 0$ because $\mathbf{b} \neq \mathbf{1}_{n}$. This proves that $\overline{\mathbf{v}}$ and $\mathbf{v}$ have the same number of nonzero components provided $\mathbf{b} \notin\left\{\mathbf{1}_{n}, \mathbf{0}_{n}\right\}$. Therefore, by Proposition 2.1(ii), we have that $\operatorname{dim}\langle\mathbf{L} ; \mathbf{b}\rangle=\operatorname{dim}\langle\mathbf{L} ; \overline{\mathbf{b}}\rangle$. 
For computational purposes, it is worth mentioning the following immediate consequence of the previous result.

Corollary 4.1: (Uncontrollable subset has even cardinality): If $n \geq 2$ then the cardinality of the set of $\{\mathbf{b} \in$ $\{0,1\}^{n} \mid(\mathbf{L}, \mathbf{b})$ is uncontrollable $\}$ is always even.

\section{A. Essentially controllable graphs}

In this section, we give a necessary condition for essential controllability. The condition depends on the following auxiliary result.

Lemma 4.1: (Order of non-identity automorphisms [20]): If all of the eigenvalues of $\mathbf{L}$ are simple then every non-identity automorphism of $\mathcal{G}$ has order two.

Proof: The proof of the claim when $\mathbf{L}$ is replaced by the adjacency matrix $\mathbf{A}$ is given in [20, Theorem 15.4]. However, the proof for the case of $\mathbf{L}$ is identical because if $\mathbf{P}$ is an automorphism of $\mathcal{G}$ then $\mathbf{P}$ commutes with both the adjacency matrix $\mathbf{A}$ and the degree matrix $\mathbf{D}$, and therefore $\mathbf{P}$ also commutes with $\mathbf{L}$.

Using the previous result, the following necessary condition for essential controllability is straightforward.

Proposition 4.2: (Essentially controllable graphs are asymmetric): Let $n \geq 3$. An essentially controllable graph on $\{0,1\}^{n}$ is asymmetric.

Proof: Let $\mathcal{G}$ be an essentially controllable graph on $\{0,1\}^{n}$. Then necessarily $\mathbf{L}$ must have distinct eigenvalues and therefore, by Lemma 4.1, every non-identity automorphism of $\mathcal{G}$ has order two. Assume by contradiction that $\mathcal{G}$ has a nontrivial automorphism group and let $\mathbf{P}$ be a permutation matrix representing a non-identity automorphism of $\mathcal{G}$. Then there exists two distinct standard basis vectors $\mathbf{e}_{i}$ and $\mathbf{e}_{j}$ such that $\mathbf{P e}_{i}=\mathbf{e}_{j}$ and $\mathbf{P} \mathbf{e}_{j}=\mathbf{e}_{i}$. Put $\mathbf{b}=\mathbf{e}_{i}+\mathbf{e}_{j}$. We note that since $n \geq 3$ we have that $\mathbf{b} \neq \mathbf{1}_{n}$. Now, $\mathbf{b}$ is clearly invariant under $\mathbf{P}$, i.e., $\mathbf{P b}=\mathbf{b}$. Thus, $\mathbf{b}$ is leader symmetric and therefore, by Proposition 3.1, ( $\mathbf{L}, \mathbf{b})$ is uncontrollable, a contradiction. This completes the proof.

According to Proposition 4.2, and since any asymmetric graph has at least six vertices [19], any essentially controllable graph has also at least six vertices. The condition given in Proposition 4.2 is, however, clearly only necessary; the graph of Figure 1(b) is an example of an asymmetric graph with six nodes that is not essentially controllable.

Example 4.1: (Essentially controllable graphs): Exactly four of the eight asymmetric graphs on six vertices are essentially controllable; these graphs are shown in Figure 2.

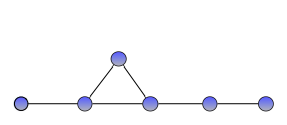

(a)

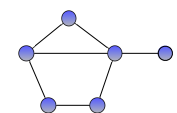

(b) (c)
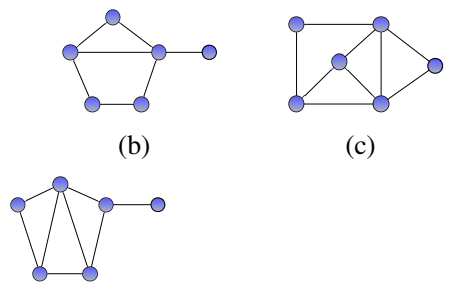

(d)

Fig. 2. All essentially controllable graphs on six vertices

In Figure 3, we display two essentially controllable graphs having orders $n=8$ and $n=11$.

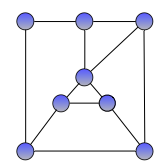

(a)

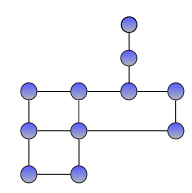

(b)
Fig. 3. Essentially controllable graphs of order $n=8$ (a), and $n=11$ (b).

The class of essentially controllable graphs are interesting for various reasons. First, this class is important from a design perspective because, except for the trivial control vectors $\mathbf{0}_{n}$ and $\mathbf{1}_{n}$, controllability is independent of the subset of nodes that receive the control inputs. This is useful when this is unknown a priori, e.g., when the control inputs are broadcasted. Another important fact about essentially controllable graphs is that the so-called minimal controllability problem is solvable [18] for these graphs. Following [18], let $B \subset \mathbb{R}^{n}$ and consider the dynamics (1) for fixed $\mathbf{b} \in \mathrm{B}$. We say that (1) is minimally controllable if $\mathbf{b}$ has the fewest number of nonzero entries among all vectors $\tilde{\mathbf{b}} \in \mathrm{B}$ such that $(\mathbf{L}, \tilde{\mathbf{b}})$ is controllable. It is shown in [18] that it is in general intractable to even approximate the number of zeros in the vector $\mathbf{b}$ that leads to minimal controllability. Nevertheless, given that the class of essentially controllable graphs are controllable using any nontrivial vector in $\{0,1\}^{n}$, the minimal controllability problem is solvable for (1) on all essentially controllable graphs, and the sparsest $\mathbf{b} \in\{0,1\}^{n}$ has $(n-1)$ nonzero entries.

We are not aware of any algorithm producing essentially controllable graphs. Given that these graphs constitute a strict subset of asymmetric graphs, and that it is NP-hard to verify if a graph has non-trivial automorphisms [23], it is unclear if the problem of generating essentially controllable graphs of order $n$ is computationally feasible. Another interesting problem is to investigate how the number of essentially controllable graphs grows, within the class of asymmetric graphs, with the number of vertices (see Table I).

\section{B. Completely uncontrollable graphs}

In this section, we study the class of completely uncontrollable graphs. Our first result shows that complete uncontrollability is not a consequence of graph symmetries. To the best of our knowledge, this important fact is overlooked in the literature on network controllability primarily because most existing results seek graph-theoretic characterizations of uncontrollability via graph symmetries. To state our first result, we need to introduce a subclass of strongly regular graphs called the block graphs of Steiner systems [24]. We begin with the following definition.

Definition 4.1: (Steiner systems): Given three integers $2 \leq$ $t<k<\nu$, a Steiner system of order $\nu$ is a pair of finite sets $(\mathcal{X}, \mathcal{B})$ where $|\mathcal{X}|=\nu$ and $\mathcal{B}$ is collection of $k$-element subsets of $\mathcal{X}$ called blocks such that every $t$-element subset of $\mathcal{X}$ is contained in one and only one block. A Steiner system will be denoted by $\mathrm{SS}(t, k, \nu)$.

Definition 4.2: (Steiner triple systems): A Steiner triple system is a $(2,3, \nu)$-Steiner system, that is, the set of blocks $\mathcal{B}$ consist of triples of $\mathcal{X}$ and every pair of points in $\mathcal{X}$ is contained in exactly one of the triples. A Steiner triple system of order $\nu$ will be denoted by $\operatorname{STS}(\nu)$. 
Example 4.2: $(\operatorname{STS}(7)):$ The sets $\mathcal{X}=\{1,2, \ldots, 7\}$ and

$$
\begin{aligned}
\mathcal{B}= & \{\{1,2,4\},\{2,3,5\},\{3,4,6\},\{4,5,7\},\{5,6,1\}, \\
& \{6,7,2\},\{7,1,3\}\}
\end{aligned}
$$

constitute a $\operatorname{STS}(7)$. This Steiner triple is called the Fano plane and is the unique Steiner triple system of order $\nu=7 . \bullet$

Example 4.3: $(\operatorname{STS}(9))$ : The sets $\mathcal{X}=\{1,2, \ldots, 9\}$ and

$$
\begin{aligned}
\mathcal{B}= & \{\{1,2,3\},\{4,5,6\},\{7,8,9\},\{1,4,7\},\{2,5,8\},\{3,6,9\} \\
& \{1,5,9\},\{2,6,7\},\{3,4,8\},\{1,6,8\},\{2,4,9\},\{3,5,7\}\}
\end{aligned}
$$

constitute a $\operatorname{STS}(9)$ and it is the unique Steiner triple system of order $\nu=9$.

The number of blocks in a $\operatorname{SS}(t, k, \nu)$ is $|\mathcal{B}|=\frac{\left(\begin{array}{c}\nu \\ t\end{array}\right)}{\left(\begin{array}{c}k \\ t\end{array}\right)}$, and in particular, a Steiner triple system of order $\nu$ contains $\nu(\nu-1) / 6$ blocks. As shown in [25], a Steiner triple system of order $\nu>1$ exists if and only if $\nu=1$ or $3(\bmod 6)$. We say that two Steiner systems $\left(\mathcal{X}_{1}, \mathcal{B}_{1}\right)$ and $\left(\mathcal{X}_{2}, \mathcal{B}_{2}\right)$ are isomorphic if there exists a bijection $\Psi: \mathcal{X}_{1} \rightarrow \mathcal{X}_{2}$ such that $\sigma \in \mathcal{B}_{1}$ if and only if $\Psi(\sigma) \in \mathcal{B}_{2}$. An automorphism of a Steiner system $(\mathcal{X}, \mathcal{B})$ is an isomorphism from $(\mathcal{X}, \mathcal{B})$ onto itself. A Steiner system is called asymmetric if it admits only the identity automorphism. It is shown in [26] that the number $N(\nu)$ of pairwise non-isomorphic Steiner triple systems of order $\nu$ satisfies $N(\nu) \geq\left(e^{-5} \nu\right)^{\nu^{2} / 12}$, and in particular, Steiner triple systems of arbitrarily large order $\nu=1$ or $3(\bmod 6)$ exist.

The block graph of a Steiner system $(\mathcal{X}, \mathcal{B})$ is the graph $\mathcal{G}_{\mathrm{SS}}$ with the blocks as vertices, that is, $\mathcal{V}=\mathcal{B}=$ $\left\{\sigma_{1}, \sigma_{2}, \ldots, \sigma_{|\mathcal{B}|}\right\}$, and the edge set consists of pairs of blocks $\left\{\sigma_{i}, \sigma_{j}\right\}$ having a nonempty intersection, i.e., $\sigma_{i} \cap \sigma_{j} \neq \emptyset$. The block graph of a Steiner triple system $\operatorname{STS}(\nu)$ is strongly regular with parameters $(n, k, \lambda, \mu)=(\nu(\nu-1) / 6,3(\nu-$ $3) / 2,(\nu+3) / 2,9)$.

Example 4.4: The block graph of $\operatorname{STS}(7)$ is the complete graph on seven vertices. The block graph of $\operatorname{STS}(9)$ is shown in Figure 4(a), and as a strongly regular graph, it has parameters $(n, k, \lambda, \mu)=(12,9,6,9)$.

Finally, it is a straightforward exercise to show that $\Psi$ : $\mathcal{X} \rightarrow \mathcal{X}$ is an automorphism of $(\mathcal{X}, \mathcal{B})$ if and only if the corresponding mapping $\Psi: \mathcal{B} \rightarrow \mathcal{B}$ is a graph automorphism of $\mathcal{G}_{\mathrm{SS}}$. With these constructions, we can now state the following result.

Theorem 4.1: (A class of completely uncontrollable asymmetric graphs): For any $K \in \mathbb{N}$ there exists a connected and asymmetric graph of order $n \geq K$ that is completely uncontrollable.

Proof: It is proved in [27, Theorem 1] that Steiner triple systems are almost always asymmetric. Explicitly, let $N(\nu)$ be the number of STSs of order $\nu$ and let $A(\nu)$ be the number of asymmetric STSs of order $\nu$. Then for $\nu=1$ or $3(\bmod 6)$ sufficiently large it holds that

$$
\frac{N(\nu)-A(\nu)}{N(\nu)}<\nu^{-\nu^{2}(1 / 16+o(1))} .
$$

In other words, the probability that a random Steiner triple system of order $\nu$ is asymmetric exceeds $1-\nu^{-\nu^{2}(1 / 16+o(1))}$. By [26], we may assume that $\nu$ is sufficiently large such that $n:=|\mathcal{B}|=\nu(\nu-1) / 6 \geq K$. Since the block graph of a Steiner triple system is strongly regular, its Laplacian matrix has only three distinct eigenvalues. The claim now follows by Proposition 2.1(ii).

It is known [28] that asymmetric Steiner triple systems of order $\nu$ exist beginning with $\nu=15$. In fact, for $\nu=15$, there are 36 asymmetric Steiner triple systems [29], one of which has blocks

$$
\begin{aligned}
& \mathcal{B}=\{\{1,2,15\},\{1,3,8\},\{1,4,5\},\{1,6,13\},\{1,7,11\}, \\
& \quad\{1,9,14\},\{1,10,12\},\{2,3,9\},\{2,4,6\},\{2,5,10\}, \\
& \quad\{2,7,13\},\{2,8,12\},\{2,11,14\},\{3,4,15\},\{3,5,11\}, \\
& \quad\{3,6,10\},\{3,7,12\},\{3,13,14\},\{4,7,10\},\{4,8,9\}, \\
& \{4,11,13\},\{4,12,14\},\{5,6,12\},\{5,7,14\},\{5,8,13\}, \\
& \{5,9,15\},\{6,7,9\},\{6,8,11\},\{6,14,15\},\{7,8,15\}, \\
& \{8,10,14\},\{9,10,13\},\{9,11,12\},\{10,11,15\},\{12,13,15\}\}
\end{aligned}
$$

and its block graph is shown in Figure 4(b).

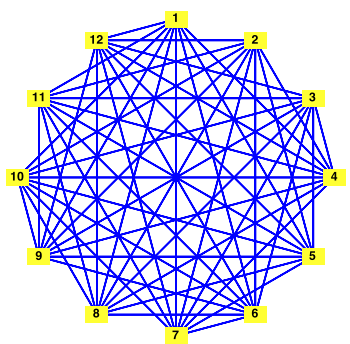

(a)

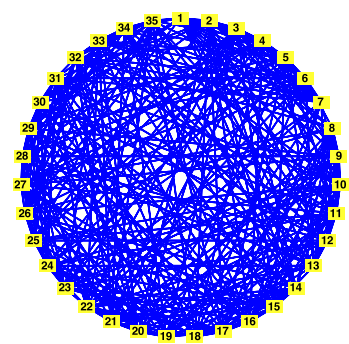

(b)
Fig. 4. (a) The block graph of $\operatorname{STS}(9)$, and (b) the block graph of an asymmetric Steiner triple system of order $\nu=15$, and as a strongly regular graph has parameters $(n, k, \lambda, \mu)=(35,18,9,9)$.

Remark 4.1: (Large asymmetric completely uncontrollable graphs): It is conjectured that almost all strongly regular graphs are asymmetric [30], and therefore all such asymmetric graphs would be completely uncontrollable. A proof of the aforementioned conjecture would provide a class of graphs larger than the block graphs of Steiner triple systems that are asymmetric and completely uncontrollable.

As shown in Theorem 4.1, uncontrollability of the block graph of a Steiner triple system is due to the Laplacian matrix having a repeated eigenvalue. It is natural then to ask if this condition is necessary for complete uncontrollability for a Laplacian-based leader-follower system. To shed light into this problem, we recall from Proposition 2.1(i) that if $\mathbf{F} \in \mathbb{R}^{n \times n}$ is diagonalizable then for any open subset $\mathrm{B} \subset \mathbb{R}^{n}$ the pair $(\mathbf{F}, \mathbf{b})$ is uncontrollable for every $\mathbf{b} \in \mathrm{B}$ if and only if $\mathbf{F}$ has a repeated eigenvalue. When $B$ is replaced by a discrete set, such as $B=\{0,1\}^{n}$, the condition of a repeated eigenvalue is no longer necessary for complete uncontrollability. For example, the symmetric matrix

$$
\mathbf{F}=\left[\begin{array}{rrrr}
2 & 0 & -1 & -1 \\
0 & 2 & -1 & -1 \\
-1 & -1 & 5 & -3 \\
-1 & -1 & -3 & 5
\end{array}\right]
$$

has distinct eigenvalues $\lambda_{1}=0, \lambda_{2}=2, \lambda_{3}=4, \lambda_{4}=8$, and it is readily verified that $(\mathbf{F}, \mathbf{b})$ is uncontrollable for every $\mathbf{b} \in\{0,1\}^{4}$. Of course, $\mathbf{F}$ is not the Laplacian matrix of any (undirected) connected graph. We have, however, verified 
numerically that for $n \in\{2,3, \ldots, 7\}$, a repeated eigenvalue is necessary and sufficient for complete uncontrollability of the Laplacian dynamics. In fact, for $n=4$ and $n=5$ we have the following, whose proof can be found in the Appendix.

Proposition 4.3: (Completely uncontrollable graphs with four and five vertices): All connected and completely uncontrollable graphs on $\{0,1\}^{4}$ and $\{0,1\}^{5}$ have a repeated eigenvalue.

However, for $n=8$ we have found ten graphs that are completely uncontrollable and have distinct eigenvalues and, for $n=9$ we have found twelve such graphs. In Figure 5 we display two such graphs on $n=8$ vertices and one for $n=9$ vertices, the latter graph being asymmetric.

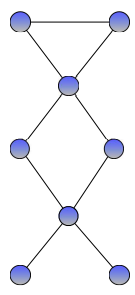

(a)

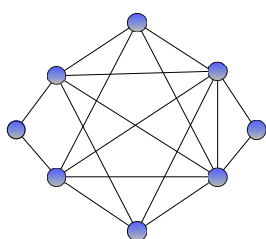

(b)

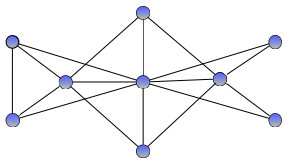

(c)
Fig. 5. (a) and (b) show two completely uncontrollable graphs with $n=8$ vertices and (c) shows a completely uncontrollable graphs with $n=9$ vertices, all with distinct eigenvalues.

Needless to say, the class of completely uncontrollable graphs with distinct eigenvalues form a very special class of graphs and have the potential to shed light on new necessary conditions for controllability and will be pursued in a future paper. For now, we focus on obtaining conditions that imply the existence of a repeated eigenvalue and consequently complete uncontrollability. To that end, we introduce the following definition.

Definition 4.3: (B-annihilators): Let $\mathrm{B} \subset \mathbb{R}^{n}$ and let $\gamma=$ $\left\{\mathbf{u}_{1}, \ldots, \mathbf{u}_{k}\right\} \subset \mathbb{R}^{n}$ be linearly independent. We say that $\gamma$ is a B-annihilator or that it annihilates $B$ if for each $\mathbf{b} \in B$ there exists $\mathbf{u}_{j} \in \gamma$ that is orthogonal to $\mathbf{b}$, that is, $\mathbf{u}_{j}^{T} \mathbf{b}=0$.

The proof of Proposition 4.3 identifies a set of three vectors that alone are $\{0,1\}^{n}$-annihilators.

Lemma 4.2: (A set of $\{0,1\}^{n}$-annihilator vectors): Let $n \geq$ 4 be a positive integer and let $\mathbf{v}_{1}, \mathbf{v}_{2}, \mathbf{v}_{3} \in \mathbb{R}^{n}$ be defined by

$$
\begin{aligned}
\mathbf{v}_{1} & =\left[\begin{array}{lllllll}
1 & -1 & 0 & 0 & 0 & \cdots & 0
\end{array}\right]^{T}, \\
\mathbf{v}_{2} & =\left[\begin{array}{lllllll}
0 & 0 & 1 & -1 & 0 & \cdots & 0
\end{array}\right]^{T}, \\
\mathbf{v}_{3} & =\left[\begin{array}{lllllll}
1 & 1 & -1 & -1 & 0 & \cdots & 0
\end{array}\right]^{T} .
\end{aligned}
$$

Then $\left\{\mathbf{v}_{1}, \mathbf{v}_{2}, \mathbf{v}_{3}\right\}$ is a $\{0,1\}^{n}$-annihilator.

Proof: Any vector $\mathbf{b} \in\{0,1\}^{n}$ having a zero in components 1 through 4 is clearly orthogonal to $\mathbf{v}_{1}$ (and $\mathbf{v}_{2}$, and $\mathbf{v}_{3}$ ). Therefore, we need only consider the binary vectors having possibly nonzero entries in components $1,2,3$, and/or 4 . There are $\sum_{k=1}^{4}\left(\begin{array}{l}4 \\ k\end{array}\right)=15$ possible cases:

i) if $\mathbf{b}=\mathbf{e}_{1}$ or $\mathbf{b}=\mathbf{e}_{2}$ then $\mathbf{b}^{T} \mathbf{v}_{2}=0$;

ii) if $\mathbf{b}=\mathbf{e}_{3}$ or $\mathbf{b}=\mathbf{e}_{4}$ then $\mathbf{b}^{T} \mathbf{v}_{1}=0$;

iii) if $\mathbf{b}(1)=\mathbf{b}(2)=1$ then $\mathbf{b}^{T} \mathbf{v}_{1}=0$;

iv) if $\mathbf{b}(1)=\mathbf{b}(3)=1$ then $\mathbf{b}^{T} \mathbf{v}_{3}=0$;

v) if $\mathbf{b}(1)=\mathbf{b}(4)=1$ then $\mathbf{b}^{T} \mathbf{v}_{3}=0$;

vi) if $\mathbf{b}(2)=\mathbf{b}(3)=1$ then $\mathbf{b}^{T} \mathbf{v}_{3}=0$; vii) if $\mathbf{b}(2)=\mathbf{b}(4)=1$ then $\mathbf{b}^{T} \mathbf{v}_{3}=0$;

viii) if $\mathbf{b}(3)=\mathbf{b}(4)=1$ then $\mathbf{b}^{T} \mathbf{v}_{2}=0$;

ix) if $\mathbf{b}(1)=\mathbf{b}(2)=\mathbf{b}(3)=1$ then $\mathbf{b}^{T} \mathbf{v}_{1}=0$;

$\mathbf{x})$ if $\mathbf{b}(1)=\mathbf{b}(2)=\mathbf{b}(4)=1$ then $\mathbf{b}^{T} \mathbf{v}_{1}=0$;

xi) if $\mathbf{b}(1)=\mathbf{b}(3)=\mathbf{b}(4)=1$ then $\mathbf{b}^{T} \mathbf{v}_{2}=0$;

xii) if $\mathbf{b}(2)=\mathbf{b}(3)=\mathbf{b}(4)=1$ then $\mathbf{b}^{T} \mathbf{v}_{2}=0$;

xiii) if $\mathbf{b}(1)=\mathbf{b}(2)=\mathbf{b}(3)=\mathbf{b}(4)=1$ then $\mathbf{b}^{T} \mathbf{v}_{3}=0$.

This ends the proof.

Next, we show that any graph containing the vectors $\left\{\mathbf{v}_{1}, \mathbf{v}_{2}, \mathbf{v}_{3}\right\}$ in (4) as eigenvectors will have a repeated eigenvalue.

Theorem 4.2: $\left(\{0,1\}^{n}\right.$-annihilator graphs and repeated eigenvalues): Let $\mathcal{G}$ be a connected graph on $n \geq 4$ vertices. If $\mathbf{v}_{1}, \mathbf{v}_{2}, \mathbf{v}_{3}$ given by (4) are eigenvectors of $\mathcal{G}$ then $\mathcal{G}$ has a repeated eigenvalue. Consequently, $\mathcal{G}$ is completely uncontrollable on $\mathbb{R}^{n}$.

Proof: Let $\mathbf{v}_{1}, \mathbf{v}_{2}, \mathbf{v}_{3}$ be eigenvectors of $\mathbf{L}$ and assume that $\mathbf{L}$ has distinct eigenvalues. Let $\left\{\mathbf{u}_{1}, \mathbf{u}_{2}, \ldots, \mathbf{u}_{n}\right\}$ be a set of orthonormal eigenvectors of $\mathbf{L}$ and, without loss of generality, let

$$
\mathbf{u}_{1}=\frac{1}{\sqrt{n}} \mathbf{1}_{n}, \mathbf{u}_{2}=\frac{1}{\sqrt{2}} \mathbf{v}_{1}, \mathbf{u}_{3}=\frac{1}{\sqrt{2}} \mathbf{v}_{2}, \mathbf{u}_{4}=\frac{1}{2} \mathbf{v}_{3} .
$$

Put $\mathbf{U}=\left[\begin{array}{llllll}\mathbf{u}_{1} & \mathbf{u}_{2} & \mathbf{u}_{3} & \mathbf{u}_{4} & \cdots & \mathbf{u}_{n}\end{array}\right]$ and let $\boldsymbol{\Lambda}=$ $\operatorname{diag}\left(\lambda_{1}, \ldots, \lambda_{n}\right)$ be the corresponding diagonal matrix of eigenvalues of $\mathbf{L}$, where $\lambda_{1}=0$ and $\lambda_{2}, \ldots, \lambda_{n}$ are in no particular order. Now, since $\mathbf{L}=\mathbf{U} \boldsymbol{\Lambda} \mathbf{U}^{T}$, a straightforward calculation shows that the upper left $4 \times 3$ submatrix of $\mathbf{L}$ is

$$
\mathbf{L}_{4 \times 3}=\left[\begin{array}{ccc}
\frac{1}{2} \lambda_{2}+\frac{1}{4} \lambda_{4} & -\frac{1}{2} \lambda_{2}+\frac{1}{4} \lambda_{4} & -\frac{1}{4} \lambda_{4} \\
-\frac{1}{2} \lambda_{2}+\frac{1}{4} \lambda_{4} & \frac{1}{2} \lambda_{2}+\frac{1}{4} \lambda_{4} & -\frac{1}{4} \lambda_{4} \\
-\frac{1}{4} \lambda_{4} & -\frac{1}{4} \lambda_{4} & \frac{1}{2} \lambda_{3}+\frac{1}{4} \lambda_{4} \\
-\frac{1}{4} \lambda_{4} & -\frac{1}{4} \lambda_{4} & -\frac{1}{2} \lambda_{3}+\frac{1}{4} \lambda_{4}
\end{array}\right] \text {. }
$$

Since $\lambda_{4}>0$, and the off diagonal entries of $\mathbf{L}$ are either 0 or -1 , we obtain from the $(1,3)$ entry of $\mathbf{L}$ that $\lambda_{4}=4$. Then, from the $(1,2)$ entry of $\mathbf{L}$, either $\lambda_{2}=2$ or $\lambda_{2}=4$. In the latter case, $\mathbf{L}$ has a repeated eigenvalue, which is a contradiction, and therefore $\lambda_{2}=2$. Similarly, from the $(4,3)$ entry of $\mathbf{L}$, the only possible cases are $\lambda_{3}=2$ or $\lambda_{3}=4$. In either case, $\mathbf{L}$ has a repeated eigenvalue, which leads to a contradiction. This completes the proof.

In Theorem 4.1, we showed the existence of (asymmetric) completely uncontrollable graphs which happened to be regular graphs. To end this section, we use Lemma 4.2 to construct a class of non-regular completely uncontrollable graphs.

Theorem 4.3: (Large uncontrollable graphs): For each $n \geq$ 6 , the set of graphs of order $n$ that are not regular and are completely uncontrollable is nonempty.

Proof: We prove the result by a direct construction. For $n=6$, consider the graph in Figure 6(a). The Laplacian matrix for this graph is

$$
\mathbf{L}_{6}=\left[\begin{array}{cccccc}
1 & -1 & 0 & 0 & 0 & 0 \\
-1 & 5 & -1 & -1 & -1 & -1 \\
0 & -1 & 3 & -1 & -1 & 0 \\
0 & -1 & -1 & 3 & 0 & -1 \\
0 & -1 & -1 & 0 & 3 & -1 \\
0 & -1 & 0 & -1 & -1 & 3
\end{array}\right]
$$


and a set of linearly independent eigenvectors of $\mathbf{L}$ are

$$
\begin{aligned}
\mathbf{u}_{1} & =\frac{1}{\sqrt{6}} \mathbf{1}_{6}^{T}, \\
\mathbf{u}_{2} & =\frac{1}{\sqrt{30}}\left[\begin{array}{llllll}
5 & -1 & -1 & -1 & -1 & -1
\end{array}\right]^{T}, \\
\mathbf{u}_{3} & =\frac{1}{\sqrt{2}}\left[\begin{array}{llllll}
0 & 0 & -1 & 0 & 0 & 1
\end{array}\right]^{T}, \\
\mathbf{u}_{4} & =\frac{1}{\sqrt{2}}\left[\begin{array}{llllll}
0 & 0 & 0 & 1 & -1 & 0
\end{array}\right]^{T}, \\
\mathbf{u}_{5} & =\frac{1}{2}\left[\begin{array}{llllll}
0 & 0 & 1 & -1 & -1 & 1
\end{array}\right]^{T}, \\
\mathbf{u}_{6} & =\frac{1}{\sqrt{20}}\left[\begin{array}{lllllll}
-4 & 0 & 1 & 1 & 1 & 1
\end{array}\right]^{T} .
\end{aligned}
$$

After a permutation of the indices, we can apply Lemma 4.2 and conclude that the set $\left\{\mathbf{u}_{3}, \mathbf{u}_{4}, \mathbf{u}_{5}\right\}$ is a $\{0,1\}^{6}$-annihilator. Now let $n \geq 6$ and extend the graph in Figure 6(a) to the graph $\mathcal{G}$ shown in Figure 6(b), where $\mathcal{G}_{n-6}$ is any connected graph on $n-6$ vertices. By construction, the Laplacian of $\mathcal{G}$ can be

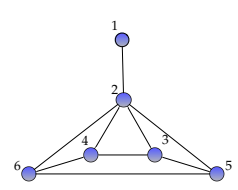

(a)

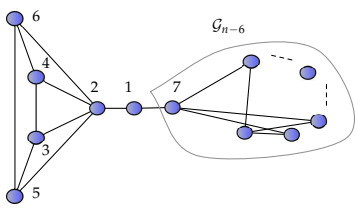

(b)
Fig. 6. A $\{0,1\}^{n}$-annihilator graph with six vertices (a), and its extension to a $\{0,1\}^{n}$-annihilator graph of any size (b).

decomposed as

$$
\mathbf{L}=\left[\begin{array}{cc}
\mathbf{L}_{6} & \mathbf{E} \\
\mathbf{E}^{T} & \mathbf{L}_{n-6}
\end{array}\right],
$$

where $\mathbf{L}_{n-6}$ denotes the Laplacian of the graph $\mathcal{G}_{n-6}$ and $\mathbf{E} \in \mathbb{R}^{6 \times(n-6)}$ is the matrix

$$
\mathbf{E}=\left[\begin{array}{llll}
-\mathbf{e}_{1} & \mathbf{0}_{n} & \cdots & \mathbf{0}_{n}
\end{array}\right]
$$

From the above decomposition of $\mathbf{L}$, and noting that the first entries of $\mathbf{u}_{3}, \mathbf{u}_{4}, \mathbf{u}_{5}$ are zero, it is not hard to see that $\mathbf{u}_{3}, \mathbf{u}_{4}$ and $\mathbf{u}_{5}$ can be lifted to eigenvectors of $\mathbf{L}$. Indeed, we have that

$$
\mathbf{L}\left[\begin{array}{c}
\mathbf{u}_{j} \\
\mathbf{0}_{n-6}
\end{array}\right]=\left[\begin{array}{c}
\mathbf{L}_{6} \mathbf{u}_{j} \\
\mathbf{0}_{n-6}
\end{array}\right]=\lambda_{j}\left[\begin{array}{c}
\mathbf{u}_{j} \\
\mathbf{0}_{n-6}
\end{array}\right] .
$$

It is clear that the lifted eigenvectors $\left[\begin{array}{c}\mathbf{u}_{j} \\ \mathbf{0}_{n-6}\end{array}\right] \in \mathbb{R}^{n}$, for $j \in$ $\{3,4,5\}$, form a set of $\{0,1\}^{n}$ annihilators. This ends the proof.

\section{Conditionally controllable graphs}

In this section we consider conditionally controllable graphs. The goal for this class would be to classify, in graphtheoretic terms, the set of control vectors $\mathbf{b} \in\{0,1\}^{n}$ such that $\operatorname{dim}\langle\mathbf{L} ; \mathbf{b}\rangle=k$, for each $k \in\{0,1,2,3, \ldots, n\}$. In other words, letting

$$
\mathcal{C}_{k}=\left\{\mathbf{b} \in\{0,1\}^{n} \mid \operatorname{dim}\langle\mathbf{L} ; \mathbf{b}\rangle=k\right\},
$$

for $k \in\{0,1,2, \ldots, n\}$, so that $\{0,1\}^{n}=\mathcal{C}_{0} \cup \mathcal{C}_{1} \cup \mathcal{C}_{2} \cup$ $\cdots \cup \mathcal{C}_{n}$ and $\mathcal{C}_{i} \cap \mathcal{C}_{j}=\emptyset$ whenever $i \neq j$, we would like to develop graph-theoretic conditions that fully characterizes $\mathcal{C}_{k}$. To this end, the main result in this section is the identification of elements in $\mathcal{C}_{2}$. It is feasible that a similar technique can be used to identify subsets of other $\mathcal{C}_{k}$ 's. We begin with the following definition.

Definition 4.4: (Homogeneous control vectors): Let $\mathcal{G}=$ $(\mathcal{V}, \mathcal{E})$ be a graph and let $\alpha, \beta \in \mathbb{N}$. We say that $\mathbf{b} \in\{0,1\}^{n}$ is a $(\alpha, \beta)$-homogeneous control vector for $\mathcal{G}$ if, for each $i \in \mathcal{V}_{\mathbf{b}}$, we have that $\alpha=\left|\mathcal{N}_{i} \cap \mathcal{V} \backslash \mathcal{V}_{\mathbf{b}}\right|$ and, for each $j \in \mathcal{V} \backslash \mathcal{V}_{\mathbf{b}}$, we have that $\beta=\left|\mathcal{N}_{j} \cap \mathcal{V}_{\mathbf{b}}\right|$. In other words, each leader node $i$ is adjacent to $\alpha$ followers and each follower node $j$ is adjacent to $\beta$ leaders.

Theorem 4.4: (Rank two control vectors): Let $\mathcal{G}$ be a graph and consider the controlled Laplacian dynamics (1), where $\mathbf{b} \in\{0,1\}^{n} \backslash\left\{\mathbf{0}_{n}, \mathbf{1}_{n}\right\}$. If $\mathbf{b}$ is a $(\alpha, \beta)$-homogeneous control vector for $\mathcal{G}$ then $\mathbf{b} \in \mathcal{C}_{2}$. In fact,

$$
\mathbf{L}^{2} \mathbf{b}=(\alpha+\beta) \mathbf{L} \mathbf{b}
$$

and therefore $\mathbf{L b}$ is an eigenvector of $\mathbf{L}$ with eigenvalue $(\alpha+$ $\beta)$. Consequently, $\operatorname{dim}\langle\mathbf{L} ; \mathbf{b}\rangle=2$. Conversely, if $\operatorname{dim}\langle\mathbf{L} ; \mathbf{b}\rangle=$ 2 then $\mathbf{L b}$ is an eigenvector of $\mathbf{L}$.

Proof: Consider the discrete linear system

$$
\mathbf{x}(k+1)=\mathbf{L x}(k),
$$

with initial condition $\mathbf{x}(0)=\mathbf{b} \in\{0,1\}^{n}$. Let $\mathbf{x}(k)=$ $\left[x_{1}(k), \cdots, \quad x_{n}(k)\right]^{T}$ denote the state vector at time $k \in$ $\mathbb{N}_{0}$. If $i \in \mathcal{V}_{\mathbf{b}}$ then $x_{i}(0)=1$ and therefore $x_{i}(1)=$ $\sum_{\ell \in \mathcal{N}_{i}}\left(x_{i}(0)-x_{\ell}(0)\right)=\alpha$. If $j \in \mathcal{V} \backslash \mathcal{V}_{\mathbf{b}}$ then $x_{j}(0)=0$ and therefore $x_{j}(1)=\sum_{\ell \in \mathcal{N}_{j}}\left(x_{j}(0)-x_{\ell}(0)\right)=-\beta$. In other words,

$$
\mathbf{L b}=\mathbf{x}(1)=\alpha \mathbf{b}+\beta\left(\mathbf{b}-\mathbf{1}_{n}\right)=(\alpha+\beta) \mathbf{b}-\beta \mathbf{1}_{n} .
$$

Then,

$$
\mathbf{L}^{2} \mathbf{b}=\mathbf{x}(2)=\mathbf{L x}(1)=(\alpha+\beta) \mathbf{L} \mathbf{b}-\beta \mathbf{L} \mathbf{1}_{n}=(\alpha+\beta) \mathbf{L} \mathbf{b}
$$

and this proves the first claim.

Now, if $\operatorname{dim}\langle\mathbf{L} ; \mathbf{b}\rangle=2$ then $\mathbf{L}^{2} \mathbf{b}=c_{0} \mathbf{b}+c_{1} \mathbf{L} \mathbf{b}$ for some $c_{0}, c_{1} \in \mathbb{R}$. Using the fact that $\mathbf{L}^{2} \mathbf{b}$ is orthogonal to $\mathbf{1}_{n}$ we immediately deduce that $c_{0}=0$. Hence, $\mathbf{L}^{2} \mathbf{b}=c_{1} \mathbf{L} \mathbf{b}$, i.e., $\mathbf{L b}$ is an eigenvector of $\mathbf{L}$. This ends the proof.

The following corollary is immediate.

Corollary 4.2: Let $\mathcal{G}$ be a connected graph on $n$-vertices and suppose that $n \geq 3$. If $\mathcal{G}$ has a $(\alpha, \beta)$-homogeneous control vector then $\mathcal{G}$ is not essentially controllable.

We give two examples that illustrate the previous result.

Example 4.5: (A rank two conditionally controllable asymmetric graph): Consider the asymmetric graph shown in Figure 7. There are 2 binary vectors $\mathbf{b}$ that result in $\operatorname{dim}\langle\mathbf{L}, \mathbf{b}\rangle=$ 2 , namely $\mathbf{b}=\mathbf{e}_{2}+\mathbf{e}_{5}$ and its binary complement $\overline{\mathbf{b}}$. For the choice $\mathbf{b}$, each control node (red nodes) is adjacent to $\alpha=2$ follower nodes (blue nodes) and each follower node is adjacent to $\beta=1$ control node. It can be verified that $\mathbf{L}^{2} \mathbf{b}=(\alpha+\beta) \mathbf{L} \mathbf{b}=3 \mathbf{L} \mathbf{b}$.

Example 4.6: (Frucht graph): As another example, consider the Frucht graph, shown in Figure 8, which is an asymmetric 3 -regular graph on $n=12$ vertices. There are 4 binary vectors $\mathbf{b}$ that result in the controllable subspace of $(\mathbf{L}, \mathbf{b})$ having dimension two, namely, $\mathbf{b}_{1}=\mathbf{e}_{1}+\mathbf{e}_{5}+\mathbf{e}_{7}+\mathbf{e}_{12}, \mathbf{b}_{2}=\mathbf{e}_{3}+\mathbf{e}_{7}+$ $\mathbf{e}_{10}$, and their binary complements $\overline{\mathbf{b}}_{1}$ and $\overline{\mathbf{b}}_{2}$, respectively. The cases $\mathbf{b}_{1}$ and $\overline{\mathbf{b}}_{1}$ are shown in Figure 8 and the cases $\mathbf{b}_{2}$ and $\overline{\mathbf{b}}_{2}$ are shown in Figure 9 . For $\mathbf{b}_{1}$, each control node 

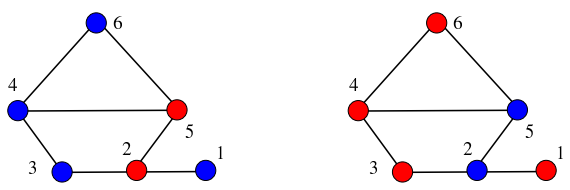

Fig. 7. Leader configuration $\mathbf{b}=\mathbf{e}_{2}+\mathbf{e}_{5}$ (left) and its binary complement $\overline{\mathbf{b}}$ (right). For b, each control node (red) is adjacent to 2 follower nodes (blue) and each follower node is adjacent to 1 control node.

(red nodes) is adjacent to $\alpha=2$ follower nodes (blue nodes) and each follower node is adjacent to $\beta=1$ control node. It can be verified that $\mathbf{L}^{2} \mathbf{b}_{1}=(\alpha+\beta) \mathbf{L} \mathbf{b}_{1}=3 \mathbf{L} \mathbf{b}_{1}$. For $\mathbf{b}_{2}$, each control node is adjacent to $\alpha=3$ follower nodes and each follower node is adjacent to $\beta=1$ control node. One can verify that $\mathbf{L}^{2} \mathbf{b}_{2}=(\alpha+\beta) \mathbf{L b}_{2}=4 \mathbf{L} \mathbf{b}_{2}$.
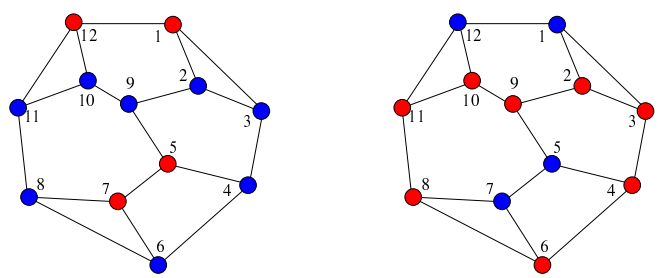

Fig. 8. Leader configuration $\mathbf{b}_{1}=\mathbf{e}_{1}+\mathbf{e}_{5}+\mathbf{e}_{7}+\mathbf{e}_{12}$ (left) and its binary complement $\overline{\mathbf{b}}_{1}$ (right). For $\mathbf{b}_{1}$, each leader node (red) is connected to 2 follower nodes (blue) and each follower is connected to 1 leader node, and vice-versa for the complement $\overline{\mathbf{b}}_{1}$.
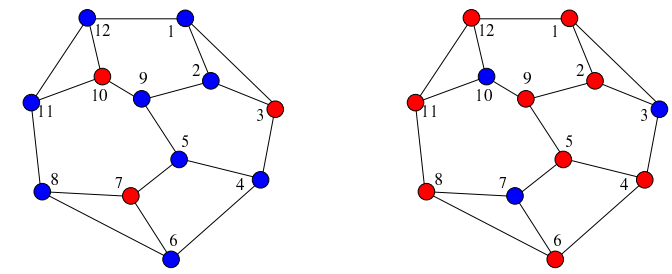

Fig. 9. Leader configuration $\mathbf{b}_{2}=\mathbf{e}_{3}+\mathbf{e}_{7}+\mathbf{e}_{10}$ (left) and its binary complement $\overline{\mathbf{b}}_{2}$ (right). For $\mathbf{b}_{2}$, each leader node (red) is connected to 3 follower nodes (blue) and each follower is connected to 1 leader nodes, and vice-versa for the complement $\overline{\mathbf{b}}_{2}$.

To end this section, we provide a lower-bound for $\operatorname{dim}\langle\mathbf{L}, \mathbf{b}\rangle$. The result depends on the following whose proof is found in the Appendix, see also [16].

Lemma 4.3: (An auxiliary result on powers of the Laplacian matrix): Let $\mathcal{G}=(\mathcal{V}, \mathcal{E})$ be a connected graph with vertex set $\mathcal{V}=\{1,2, \ldots, n\}$, and let $r \in \mathbb{N}$. Then for $i, j \in \mathcal{V}$ such that $r \leq \mathrm{d}_{\mathcal{G}}(i, j)$ we have that $\left(\mathbf{L}^{k}\right)_{i j}=0$, for all $0 \leq k<r$, and

$$
\left(\mathbf{L}^{r}\right)_{i j}=(-1)^{r}\left(\mathbf{A}^{r}\right)_{i j}
$$

To state our lower bound, we need a further piece of notation. Given a follower node $j \in \mathcal{V} \backslash \mathcal{V}_{\mathbf{b}}$, we denote by $r_{j}$ the minimum distance of $j$ to the set of control nodes, that is,

$$
r_{j}=\min _{i \in \mathcal{V}_{\mathbf{b}}} \mathrm{d}_{\mathcal{G}}(i, j) .
$$

We define the control diameter of $\mathbf{b}$ by

$$
r_{\mathbf{b}}=\max _{j \in \mathcal{V} \backslash \mathcal{V}_{\mathbf{b}}} r_{j}
$$

The following result appears in [17] for the single-leader case and in [16] for the multiple-leader case. To keep this paper self-contained, we include its short proof.

Theorem 4.5: (A lower-bound on the rank of the controllability matrix): Let $\mathcal{G}=(\mathcal{V}, \mathcal{E})$ be a connected graph and consider the Laplacian dynamics (1), where $\mathbf{b} \in$ $\{0,1\}^{n} \backslash\left\{\mathbf{1}_{n}, \mathbf{0}_{n}\right\}$. Then

$$
\operatorname{dim}\langle\mathbf{L} ; \mathbf{b}\rangle \geq r_{\mathbf{b}}+1
$$

where $r_{\mathbf{b}}$ is the control radius of $\mathbf{b}$.

Proof: For a follower node $j \in \mathcal{V} \backslash \mathcal{V}_{\mathbf{b}}$ let $K_{j}:=\{i \in$ $\left.\mathcal{V}_{\mathbf{b}} \mid \mathrm{d}_{\mathcal{G}}(i, j)=r_{j}\right\}$. From Lemma 4.3, and linearity, it follows that $\left(\mathbf{L}^{k} \mathbf{b}\right)_{j}=0$ for all $0 \leq k<r_{j}$ and

$$
\left(\mathbf{L}^{r_{j}} \mathbf{b}\right)_{j}=(-1)^{r_{j}} \sum_{i \in K_{j}}\left(\mathbf{A}^{r_{j}}\right)_{i j} .
$$

It is well-known that $\left(\mathbf{A}^{k}\right)_{i j}$ is the number of walks from $i$ to $j$ of length $k$ [20,pg. 11]. Hence, by definition of $r_{j}$, we have $\left(\mathbf{A}^{r_{j}}\right)_{i j}>0$ for all $i \in K_{j}$. This implies that $\left(\mathbf{L}^{r_{j}} \mathbf{b}\right)_{j} \neq 0$. It follows that $\left\{\mathbf{b}, \mathbf{L b}, \ldots, \mathbf{L}^{r_{j}} \mathbf{b}\right\}$ is a linearly independent set of vectors. The claim follows by taking $r_{j}=r_{\mathbf{b}}$.

Using Theorem 4.3, we obtain an alternative proof of the following known fact about the controllability of a path graph.

Corollary 4.3: (Controllability of path graphs [7]): The path graph $P_{n}$ is controllable when the leader node is chosen as one of the terminal nodes.

$$
\text { Proof: Let } \mathcal{G} \quad=\quad \begin{array}{r}
P_{n} \\
=
\end{array}
$$
$\{\{1,2, \ldots, n\},\{\{1,2\},\{2,3\}, \ldots,\{n-1, n\}\} \quad$ be the path graph of length $n$. If $\mathbf{b}=\mathbf{e}_{n}$ then the control radius is $r_{\mathbf{b}}=\mathrm{d}_{\mathcal{G}}(1, n)=n-1$, and therefore by Theorem 4.5 we must have that $\operatorname{dim}\langle\mathbf{L} ; \mathbf{b}\rangle=n$, i.e., $(\mathbf{L}, \mathbf{b})$ is controllable.

\section{Enumeration of Controllability Classes for Small Graphs}

In this section we provide numerical results on the cardinality of the controllability classes. In Table I, we enumerate the graph controllability classes for small connected graphs from order $n=2$ through $n=9$. In the table, $g_{n}$ is the number of connected graphs, $a_{n}$ is the number of asymmetric connected graphs, $e_{n}$ is the number of essentially controllable graphs, $u_{n}$ is the number of completely uncontrollable graphs, and $c_{n}$ is the number of conditionally controllable graphs, where $n$ is the order of the graph.

\begin{tabular}{cccccc}
\hline \hline$n$ & $g_{n}$ & $a_{n}$ & $e_{n}$ & $u_{n}$ & $c_{n}$ \\
\hline \hline 2 & 1 & 0 & 1 & 0 & 0 \\
3 & 2 & 0 & 0 & 1 & 1 \\
4 & 6 & 0 & 0 & 4 & 2 \\
5 & 21 & 0 & 0 & 11 & 10 \\
6 & 112 & 8 & 4 & 59 & 49 \\
7 & 853 & 144 & 84 & 264 & 505 \\
8 & 11117 & 3552 & 1992 & 2764 & 6361 \\
9 & 261080 & 131452 & 94084 & 29750 & 137246 \\
\hline \hline
\end{tabular}

TABLE I

ENUMERATION OF CONTROLLABILITY CLASSES FOR SMALL GRAPHS

Values of the sequences $g_{n}$ and $a_{n}$ can be found in [31]. We used Maple's Graph Theory package to generate the adjacency matrices of all connected graphs on $2 \leq n \leq 9$ vertices. The data in Table I suggests that the ratio $e_{n} / a_{n}$ is growing with $n$. It is an interesting problem to investigate if almost all 
asymmetric graphs are essentially controllable on $\{0,1\}^{n}$ as $n \rightarrow \infty$.

\section{CONCLUSiOn AND Future WORK}

We have considered the controllability problem for the Laplacian-based leader-follower dynamics. We introduced the class of essentially controllable, completely uncontrollable, and conditionally controllable graphs. We proved that the set of essentially controllable graphs is strict subset of the set of asymmetric graphs and classified all essentially controllable graphs on six vertices. We provided a class of asymmetric completely uncontrollable graphs, namely the block graphs of Steiner triple systems. We proved that for connected graphs with four or five vertices, having a repeated eigenvalue fully characterizes complete uncontrollability. We gave a sufficient condition for complete uncontrollability in terms of the eigenvectors of the Laplacian matrix, which also leads to repeated eigenvalues. We have also shown the existence of completely uncontrollable graphs with distinct eigenvalues for graphs with eight vertices and higher. We identified a class of homogeneous binary control vectors that result in a twodimensional controllable subspace. Finally, we gave a lowerbound on the dimension of the controllable subspace in terms of the distance of the followers to the leaders.

There are several natural open problems we plan to investigate further. The characterization of the graphs that are completely uncontrollable and have distinct eigenvalues is a very interesting problem. From a design perspective, the class of essentially controllable graphs are robust to the choice of input vertices; hence finding sufficient conditions for an asymmetric graph to be essentially controllable and investigating if the class of essentially controllable graphs asymptotically approaches the class of asymmetric graphs are of great importance. Investigating the existence of a polynomial-time algorithm for generating essentially controllable graphs, exploring scenarios with multiple leaders, and extending the proposed classifications to other, possibly nonlinear, networked control systems are other areas of future work.

\section{REFERENCES}

[1] C. Aguilar and B. Gharesifard, "A graph-theoretic classification for the controllability of the laplacian leader-follower dynamics," in IEEE Conf. on Decision and Control, (Los Angles, CA), 2014. submitted.

[2] M. Mesbahi and M. Egerstedt, Graph Theoretic Methods in Multiagent Networks. Applied Mathematics Series, Princeton University Press, 2010.

[3] W. Ren and Y. Cao, Distributed Coordination of Multi-Agent Networks. Communications and Control Engineering, New York: Springer, 2011.

[4] F. Bullo, J. Cortés, and S. Martínez, Distributed Control of Robotic Networks. Applied Mathematics Series, Princeton University Press, 2009. Electronically available at http://coordinationbook.info.

[5] M. O. Jackson, Social and Economic Networks. Princeton University Press, 2010.

[6] C.-T. Lin, "Structural controllability," IEEE Transactions on Automatic Control, vol. 19, no. 3, pp. 201-208, 1974.

[7] H. Tanner, "On the controllability of nearest neighbor interconnections," in IEEE Conf. on Decision and Control, pp. 2467-2472, 2004.

[8] A. Rahmani, M. Ji, M. Mesbahi, and M. Egerstedt, "Controllability of multi-agent systems from a graph-theoretic perspective," SIAM, vol. 48 , no. 1, pp. 162-186, 2009.

[9] Z. Ji, Z. Wang, H. Lin, and Z. Wang, "Interconnection topologies for multi-agent coordination under leader-follower framework," Automatica, vol. 45, pp. 2857-2863, 2009.
[10] Z. Ji, H. Lin, and H. Yu, "Leaders in multi-agent controllability under consensus algorithm and tree topology," Systems Control Letters, vol. 61, pp. 918-925, 2012.

[11] G. Parlangeli and G. Notarstefano, "On the reachability and observability of path and cycle graphs," IEEE Trans. Autom. Control, vol. 57, no. 3, pp. 743-748, 2012.

[12] G. Notarstefano and G. Parlangeli, "Controllability and observability of grid graphs via reduction of symmetries," IEEE Trans. Autom. Control, vol. 58, no. 7, pp. 1719-1731, 2013.

[13] D. Cvetković, P. Rowlinson, Z. Stanić, and M.-G. Yoon, "Controllable graphs," Bull. Acad. Serbe Sci. Arts. Classe Sci. Math. Natur. Sci. Math., vol. 140, no. 36, pp. 81-88, 2011.

[14] D. Cvetković, P. Rowlinson, Z. Stanić, and M.-G. Yoon, "Controllable graphs with least eigenvalue at least -2," Appl. Anal. Discrete Math., vol. 5, pp. 165-175, 2011.

[15] C. Godsil, "Controllable subsets in graphs," Ann. Comb., vol. 16, pp. 733-744, 2012.

[16] A. Yazicioglu, W. Abbas, and M. Egerstedt, "A tight lower bound on the controllability of networks with multiple leaders," in IEEE Conf. on Decision and Control, pp. 1978-1983, 2012.

[17] S. Zhang, M. Camlibel, and M. Cao, "Controllability of diffusivelycoupled multi-agent systems with general and distance regular coupling topologies," in IEEE Conf. on Decision and Control, pp. 759-764, 2011.

[18] A. Olshevsky, "Minimal controllability problems," preprint, 2014. http://arxiv.org/abs/1304.3071.

[19] P. Erdös and A. Réyni, "Asymmetric graphs," Acta Math. Acad. Sci. Hungary, vol. 14, pp. 295-315, 1963.

[20] N. Biggs, Algebraic Graph Theory. Cambridge University Press, 1974

[21] R. Diestel, Graph Theory. Springer-Verlag New York, Inc., 1997.

[22] C. Godsil and R. Gordon, Algebraic Graph Theory. Springer, 2001.

[23] A. Lubiw, "Some np-complete problems similar to graph isomorphism," SIAM Journal on Computing, vol. 10, no. 1, pp. 11-21, 1982.

[24] C. Colbourn and A. Rosa, Triple Systems. Clarendon Press Oxford, 1999.

[25] T. Kirkman, "On a problem in combinatorics," Cambridge Dublin Math. J., vol. 2, pp. 191-204, 1847.

[26] R. Wilson, "Nonisomorphic steiner triple systems," Mathematische Zeitschrift, vol. 135, pp. 303-313, 1974.

[27] L. Babai, "Almost all Steiner triple systems are asymmetric," Annals of Discrete Mathematics, vol. 7, pp. 37-39, 1980.

[28] C. Linder and A. Rosa, "On the existence of automorphism free steiner triple systems," Journal of Algebra, vol. 34, pp. 430-443, 1975.

[29] R. Fisher, "An examination of the different possible solutions of a problem in incomplete blocks," Annals of Human Genetics, vol. 10, pp. 52-75, 1940.

[30] L. Babai, "Combinatorial optimization," in Handbook of Combinatorics, Volume II, Chapter 27 (R. L. Graham, M. Grötschel, and L. Lovász, eds.), Elsevier (North-Holland), 1995.

[31] N. Sloane and S. Plouffe, The Encyclopedia of Integer Sequences. Academic Press, 1995.

\section{APPENDIX}

\section{A. Proof of Proposition 3.1}

Here, we provide an alternative proof of Proposition 3.1. The proof is an easy consequence of the following result.

Lemma A.1: (Invariance of graph automorphisms under the Laplacian matrix): If $\mathbf{P}$ is an automorphism of $\mathcal{G}$ and $\mathbf{P} \mathbf{v}=\mathbf{v}$ for some $\mathbf{v} \in \mathbb{R}^{n}$ then $\mathbf{P L v}=\mathbf{L v}$. In other words, the subspace of fixed elements of $\mathbf{P}$ is invariant under the Laplacian matrix $\mathbf{L}$.

Proof: If $\mathbf{P}$ is an automorphism of $\mathcal{G}$ then $\mathbf{P}$ commutes with both the adjacency matrix $\mathbf{A}$ and the degree matrix $\mathbf{D}$, and therefore it also commutes with the Laplacian matrix $\mathbf{L}$. Therefore, $\mathbf{P L v}=\mathbf{L P v}=\mathbf{L v}$.

We now prove Proposition 3.1.

Proof of Proposition 3.1: If $\mathbf{b}$ is leader symmetric then there exists an automorphism $\mathbf{P}$ such that $\mathbf{P b}=\mathbf{b}$. Applying Lemma A.1 recursively we have that $\mathbf{P} \mathbf{L}^{k} \mathbf{b}=\mathbf{L}^{k} \mathbf{b}$ for any $k \in \mathbb{N}_{0}$. Thus, the subspace $\langle\mathbf{L} ; \mathbf{b}\rangle$ lies in the eigenspace of $\mathbf{P}$ associated to the eigenvalue $\lambda=1$. Because $\mathbf{P}$ is not the 
identity matrix it follows that $\langle\mathbf{L} ; \mathbf{b}\rangle$ is a strict subspace of $\mathbb{R}^{n}$.

\section{B. Proof of Proposition 4.3}

Case $n=4$ : Let $\mathbf{L}$ be the Laplacian matrix of a connected graph that is completely uncontrollable on $\{0,1\}^{4}$. Suppose by contradiction that $\mathbf{L}$ has distinct eigenvalues $0=\lambda_{1}, \lambda_{2}, \lambda_{3}, \lambda_{4}$. Then any basis of $\mathbb{R}^{4}$ of eigenvectors of $\mathbf{L}$ is uniquely determined up to scalar multiples. Let $\left\{\mathbf{u}_{1}, \mathbf{u}_{2}, \mathbf{u}_{3}, \mathbf{u}_{4}\right\}$ be basis of $\mathbb{R}^{4}$ consisting of mutually orthogonal eigenvectors of $\mathbf{L}$. Then by Proposition 2.1(ii), the basis $\left\{\mathbf{u}_{1}, \mathbf{u}_{2}, \mathbf{u}_{3}, \mathbf{u}_{4}\right\}$ annihilates $\{0,1\}^{4}$. Without loss of generality (w.l.o.g.), let $\mathbf{u}_{1}=[1,1,1,1]^{T}$. Then $\left\{\mathbf{u}_{2}, \mathbf{u}_{3}, \mathbf{u}_{4}\right\}$ annihilates the standard basis vectors $\left\{\mathbf{e}_{1}, \mathbf{e}_{2}, \mathbf{e}_{3}, \mathbf{e}_{4}\right\} \subset\{0,1\}^{4}$. Therefore, by the pigeon hole principle, one of the vectors in $\left\{\mathbf{u}_{2}, \mathbf{u}_{3}, \mathbf{u}_{4}\right\}$, say $\mathbf{u}_{2}$, must contain two zero entries. Then, since $\mathbf{u}_{2} \perp \mathbf{u}_{1}$, we have (possibly after permuting coordinates) that $\mathbf{u}_{2}=[1,-1,0,0]^{T}$. Without loss of generality, we may now assume that $\mathbf{u}_{3} \perp \mathbf{e}_{1}$, and since $\mathbf{u}_{3} \perp\left\{\mathbf{u}_{1}, \mathbf{u}_{2}\right\}$, it follows that $\mathbf{u}_{3}$ is of the form $\mathbf{u}_{3}=[0,0,1,-1]^{T}$. Finally, since $\mathbf{u}_{4} \perp\left\{\mathbf{u}_{1}, \mathbf{u}_{2}, \mathbf{u}_{3}\right\}$, then $\mathbf{u}_{4}=[1,1,-1,-1]^{T}$. Now put,

$$
\mathbf{U}=\left[\begin{array}{llll}
\frac{1}{\left\|\mathbf{u}_{1}\right\|} \mathbf{u}_{1} & \frac{1}{\left\|\mathbf{u}_{2}\right\|} \mathbf{u}_{2} \quad \frac{1}{\left\|\mathbf{u}_{3}\right\|} \mathbf{u}_{3} \quad \frac{1}{\left\|\mathbf{u}_{4}\right\|} \mathbf{u}_{4}
\end{array}\right] \text {. }
$$

Then, since $\mathbf{L}=\mathbf{U} \operatorname{diag}\left(\left[0, \lambda_{2}, \lambda_{3}, \lambda_{4}\right]\right) \mathbf{U}^{T}$, we obtain that

$$
\begin{gathered}
\mathbf{L}=\left[\begin{array}{ll}
\mathbf{X}_{1} & \mathbf{X}_{2} \\
\mathbf{X}_{2}^{T} & \mathbf{X}_{3}
\end{array}\right], \\
\mathbf{X}_{1}=\left[\begin{array}{cc}
\lambda_{3}+\frac{1}{4} \lambda_{4} & -\frac{1}{2} \lambda_{3}+\frac{1}{4} \lambda_{4} \\
-\frac{1}{2} \lambda_{3}+\frac{1}{4} \lambda_{4} & \frac{1}{2} \lambda_{3}+\frac{1}{4} \lambda_{4}
\end{array}\right], \\
\mathbf{X}_{2}=-\frac{\lambda_{4}}{4}\left[\begin{array}{cc}
1 & 1 \\
1 & 1
\end{array}\right], \\
\mathbf{X}_{3}=\left[\begin{array}{cc}
\frac{1}{2} \lambda_{2}+\frac{1}{4} \lambda_{4} & -\frac{1}{2} \lambda_{2}+\frac{1}{4} \lambda_{4} \\
-\frac{1}{2} \lambda_{2}+\frac{1}{4} \lambda_{4} & \frac{1}{2} \lambda_{2}+\frac{1}{4} \lambda_{4}
\end{array}\right] .
\end{gathered}
$$

Since $\lambda_{4}>0$, and the off diagonal entries of $\mathbf{L}$ are either 0 or -1 , we obtain from the $(1,3)$ entry of $\mathbf{L}$ that $\lambda_{4}=4$. Then, from the $(1,2)$ entry of $\mathbf{L}$, either $\lambda_{3}=2$ or $\lambda_{3}=4$. In the latter case, $\mathbf{L}$ has a repeated eigenvalue, which is a contradiction, and therefore $\lambda_{3}=2$. Then, from the $(3,4)$ entry of $\mathbf{L}$, the only possible cases are $\lambda_{2}=2$ or $\lambda_{2}=4$. In either case, $\mathbf{L}$ has a repeated eigenvalue, which leads to a contradiction. This completes the proof for $n=4$.

Case $n=5$ : Let $\mathbf{L}$ be the Laplacian matrix of a connected graph that is completely uncontrollable on $\{0,1\}^{5}$. Suppose by contradiction that $\mathbf{L}$ has distinct eigenvalues $0=\lambda_{1}, \lambda_{2}, \lambda_{3}, \lambda_{4}, \lambda_{5}$. Then, by Proposition 2.1(ii), there exists an orthogonal set $\left\{\mathbf{u}_{1}, \mathbf{u}_{2}, \mathbf{u}_{3}, \mathbf{u}_{4}, \mathbf{u}_{5}\right\}$ of eigenvectors of $\mathbf{L}$ that annihilates $\{0,1\}^{5}$. Without loss of generality, let $\mathbf{u}_{1}=\left[\begin{array}{lllll}1 & 1 & 1 & 1 & 1\end{array}\right]^{T}$. Then $\left\{\mathbf{u}_{2}, \mathbf{u}_{3}, \mathbf{u}_{4}, \mathbf{u}_{5}\right\}$ annihilates the set $S_{2}=\left\{\mathbf{b} \in\{0,1\}^{5} \mid\|\mathbf{b}\|_{1}=2\right\}$, i.e., the set of elements in $\{0,1\}^{5}$ containing two nonzero entries. Now $\left|S_{2}\right|=\left(\begin{array}{l}5 \\ 2\end{array}\right)=10$, and therefore by the pigeon hole principle, there is at least one vector in $\left\{\mathbf{u}_{2}, \mathbf{u}_{3}, \mathbf{u}_{4}, \mathbf{u}_{5}\right\}$, say $\mathbf{u}_{2}$, that is orthogonal to at least three vectors in $S_{2}$. Hence, let $\mathbf{b}_{1}, \mathbf{b}_{2}, \mathbf{b}_{3} \in S_{2}$ be distinct vectors such that $\mathbf{u}_{2} \perp\left\{\mathbf{b}_{1}, \mathbf{b}_{2}, \mathbf{b}_{3}\right\}$. There are three cases to consider.
Case 1. Suppose that the number of distinct indices where $\mathbf{b}_{1}, \mathbf{b}_{2}, \mathbf{b}_{3}$ are nonzero is three. Say $\mathbf{b}_{1}$ is nonzero at the pair of indices $(i, j), \mathbf{b}_{2}$ is nonzero at the pair of indices $(j, k)$, and thus $\mathbf{b}_{3}$ is nonzero at the pair of indices $(i, k)$. Then from $\mathbf{u}_{2} \perp\left\{\mathbf{b}_{1}, \mathbf{b}_{2}, \mathbf{b}_{3}\right\}$ we obtain

$$
\left[\begin{array}{lll}
1 & 1 & 0 \\
0 & 1 & 1 \\
1 & 0 & 1
\end{array}\right]\left[\begin{array}{l}
\mathbf{u}_{2}(i) \\
\mathbf{u}_{2}(j) \\
\mathbf{u}_{2}(k)
\end{array}\right]=\left[\begin{array}{l}
0 \\
0 \\
0
\end{array}\right],
$$

whose unique solution is $\mathbf{u}_{2}(i)=\mathbf{u}_{2}(j)=\mathbf{u}_{2}(k)=0$. Therefore, since $\mathbf{u}_{2} \perp \mathbf{u}_{1}$ we have (possibly after permuting indices) that $\mathbf{u}_{2}=\left[\begin{array}{lllll}1 & -1 & 0 & 0 & 0\end{array}\right]$. Now, $\mathbf{u}_{2}$ is orthogonal to $\mathbf{b}_{1}=\left[\begin{array}{lllll}0 & 0 & 1 & 1 & 0\end{array}\right]^{T}, \mathbf{b}_{2}=\left[\begin{array}{lllll}0 & 0 & 1 & 0 & 1\end{array}\right]^{T}, \mathbf{b}_{3}=\left[\begin{array}{lllll}0 & 0 & 0 & 1 & 1\end{array}\right]^{T}$, and also $\mathbf{b}_{4}=\left[\begin{array}{lllll}1 & 1 & 0 & 0 & 0\end{array}\right]^{T}$. This leaves the following six vectors in $S_{2}$ that are annihilated by $\left\{\mathbf{u}_{3}, \mathbf{u}_{4}, \mathbf{u}_{5}\right\}$ :

$$
\begin{aligned}
& \mathbf{b}_{5}=\left[\begin{array}{lllll}
1 & 0 & 1 & 0 & 0
\end{array}\right]^{T}, \mathbf{b}_{6}=\left[\begin{array}{lllll}
1 & 0 & 0 & 1 & 0
\end{array}\right]^{T}, \mathbf{b}_{7}=\left[\begin{array}{lllll}
1 & 0 & 0 & 0 & 1
\end{array}\right]^{T}, \\
& \mathbf{b}_{8}=\left[\begin{array}{lllll}
0 & 1 & 1 & 0 & 0
\end{array}\right]^{T}, \mathbf{b}_{9}=\left[\begin{array}{lllll}
0 & 1 & 0 & 1 & 0
\end{array}\right]^{T}, \mathbf{b}_{10}=\left[\begin{array}{lllll}
0 & 1 & 0 & 0 & 1
\end{array}\right]^{T} .
\end{aligned}
$$

Now, we may assume that $\mathbf{u}_{3} \perp \mathbf{e}_{1}$, and since also $\mathbf{u}_{3} \perp$

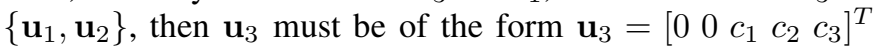
where $c_{1}+c_{2}+c_{3}=0$. Now, by the pigeon hole principle, $\mathbf{u}_{3}$ is orthogonal to at least two vectors in $\left\{\mathbf{b}_{5}, \ldots, \mathbf{b}_{10}\right\}$. We claim that in fact $\mathbf{u}_{3}$ is orthogonal to exactly two of them. Indeed, suppose that $\mathbf{u}_{3} \perp \mathbf{b}_{5}$. Then clearly $c_{1}=0$ and thus $c_{2}=-c_{3} \neq 0$. Now, in this case we also have that $\mathbf{u}_{3} \perp \mathbf{b}_{8}$. If $\mathbf{u}_{3} \perp \mathbf{b}_{6}$ or $\mathbf{u}_{3} \perp \mathbf{b}_{7}$ then clearly $c_{2}=c_{3}=0$, which is a contradiction since $\mathbf{u}_{3} \neq \mathbf{0}$. Similarly, if $\mathbf{u}_{3} \perp \mathbf{b}_{9}$ or $\mathbf{u}_{3} \perp \mathbf{b}_{10}$ then again $c_{2}=c_{3}=0$, which is a contradiction. Thus, if $\mathbf{u}_{3} \perp \mathbf{b}_{5}$ then $\mathbf{u}_{3} \perp \mathbf{b}_{8}$, and $\mathbf{u}_{3}$ is not orthogonal to $\left\{\mathbf{b}_{6}, \mathbf{b}_{7}, \mathbf{b}_{8}, \mathbf{b}_{9}, \mathbf{b}_{10}\right\}$. Similar arguments show that if $\mathbf{u}_{3} \perp \mathbf{b}_{6}$ then $\mathbf{u}_{3} \perp \mathbf{b}_{9}$, and $\mathbf{u}_{3}$ is not orthogonal to any vector in $\left\{\mathbf{b}_{6}, \mathbf{b}_{7}, \mathbf{b}_{8}, \mathbf{b}_{10}\right\}$, and that if $\mathbf{u}_{3} \perp \mathbf{b}_{7}$ then $\mathbf{u}_{3} \perp \mathbf{b}_{10}$, and $\mathbf{u}_{3}$ is not orthogonal to any vector in $\left\{\mathbf{b}_{5}, \mathbf{b}_{6}, \mathbf{b}_{8}, \mathbf{b}_{9}\right\}$. Hence, after a possible permutation of the indices, we have that $\mathbf{u}_{3}=[0,0,0,1,-1]^{T}$, and therefore $\mathbf{u}_{3} \perp\left\{\mathbf{b}_{5}, \mathbf{b}_{8}\right\}$. Now, since $\mathbf{u}_{4} \perp\left\{\mathbf{u}_{1}, \mathbf{u}_{2}, \mathbf{u}_{3}\right\}$, it follows that $\mathbf{u}_{4}$ takes the form $\mathbf{u}_{4}=(a, a,-2(a+b), b, b)$ for $a, b \neq 0$. Now, $\mathbf{u}_{4}$ is orthogonal to one of $\mathbf{b}_{6}, \mathbf{b}_{7}, \mathbf{b}_{9}, \mathbf{b}_{10}$. It is readily verified that in any case this implies that $a=-b$. Therefore, we have that $\mathbf{u}_{4}=\left[\begin{array}{lllll}1 & 1 & 0 & -1 & -1\end{array}\right]^{T}$, and this implies that $\mathbf{u}_{5}=\left[\begin{array}{lllll}1 & 1 & -4 & 1 & 1\end{array}\right]^{T}$. Now put

$$
\mathbf{U}=\left[\begin{array}{ccccc}
\frac{1}{\left\|\mathbf{u}_{1}\right\|} \mathbf{u}_{1} & \frac{1}{\left\|\mathbf{u}_{2}\right\|} \mathbf{u}_{2} & \frac{1}{\left\|\mathbf{u}_{3}\right\|} \mathbf{u}_{3} & \frac{1}{\left\|\mathbf{u}_{4}\right\|} \mathbf{u}_{4} & \frac{1}{\left\|\mathbf{u}_{5}\right\|} \mathbf{u}_{5}
\end{array}\right]
$$

and compute $\mathbf{L}=\mathbf{U d i a g}\left(\left[0, \lambda_{2}, \lambda_{3}, \lambda_{4}, \lambda_{5}\right]\right) \mathbf{U}^{T}$. The first column of $\mathbf{L}$ is

$$
\mathbf{L}_{1}=\left[\begin{array}{c}
\frac{1}{2} \lambda_{2}+\frac{1}{4} \lambda_{4}+\frac{1}{20} \lambda_{5} \\
-\frac{1}{2} \lambda_{2}+\frac{1}{4} \lambda_{4}+\frac{1}{20} \lambda_{5} \\
-\frac{1}{5} \lambda_{5} \\
-\frac{1}{4} \lambda_{4}+\frac{1}{20} \lambda_{5} \\
-\frac{1}{4} \lambda_{4}+\frac{1}{20} \lambda_{5}
\end{array}\right]
$$

From the third component of $\mathbf{L}_{1}$, we see that because $0<\lambda_{5}$ and the off diagonal entries of $\mathbf{L}$ are either 0 or -1 , we must have $\lambda_{5}=5$. The updated $\mathbf{L}$ is

$$
\mathbf{L}=\left[\begin{array}{ll}
\mathbf{X}_{1} & \mathbf{X}_{2} \\
\mathbf{X}_{2}^{T} & \mathbf{X}_{3}
\end{array}\right]
$$


where

$$
\begin{aligned}
& \mathbf{X}_{1}=\left[\begin{array}{ccc}
\frac{1}{2} \lambda_{2}+\frac{1}{4} \lambda_{4}+\frac{1}{4} & -\frac{1}{2} \lambda_{2}+\frac{1}{4} \lambda_{4}+\frac{1}{4} & -1 \\
-\frac{1}{2} \lambda_{2}+\frac{1}{4} \lambda_{4}+\frac{1}{4} & \frac{1}{2} \lambda_{2}+\frac{1}{4} \lambda_{4}+\frac{1}{4} & -1 \\
-1 & -1 & 4
\end{array}\right], \\
& \mathbf{X}_{2}=\frac{1}{4}\left(1-\lambda_{4}\right)\left[\begin{array}{cc}
1 & 1 \\
1 & 1
\end{array}\right], \\
& \mathbf{X}_{3}=\left[\begin{array}{cc}
\frac{1}{2} \lambda_{3}+\frac{1}{4} \lambda_{4}+\frac{1}{4} & -\frac{1}{2} \lambda_{3}+\frac{1}{4} \lambda_{4}+\frac{1}{4} \\
-\frac{1}{2} \lambda_{3}+\frac{1}{4} \lambda_{4}+\frac{1}{4} & \frac{1}{2} \lambda_{3}+\frac{1}{4} \lambda_{4}+\frac{1}{4}
\end{array}\right] .
\end{aligned}
$$

Consider the $(1,5)$ entry of $\mathbf{L}$, namely $-\frac{1}{4} \lambda_{4}+\frac{1}{4}$. The only possible cases are that $\lambda_{4}=5$ or $\lambda_{4}=1$. In the case $\lambda_{4}=$ 5 we have a repeated eigenvalue, which is a contradiction. Therefore, $\lambda_{4}=1$, and the updated Laplacian matrix is

$$
\begin{gathered}
\mathbf{L}=\left[\begin{array}{cc}
\mathbf{X}_{1} & \mathbf{X}_{2} \\
\mathbf{X}_{2}^{T} & \mathbf{X}_{3}
\end{array}\right], \quad \text { where } \\
\mathbf{X}_{1}=\left[\begin{array}{ccc}
\frac{1}{2} \lambda_{2}+\frac{1}{2} & -\frac{1}{2} \lambda_{2}+\frac{1}{2} & -1 \\
-\frac{1}{2} \lambda_{2}+\frac{1}{2} & \frac{1}{2} \lambda_{2}+\frac{1}{2} & -1 \\
-1 & -1 & 4
\end{array}\right], \\
\mathbf{X}_{2}=\mathbf{0}_{2 \times 2}, \\
\mathbf{X}_{3}=\left[\begin{array}{cc}
\frac{1}{2} \lambda_{3}+\frac{1}{2} & -\frac{1}{2} \lambda_{3}+\frac{1}{2} \\
-\frac{1}{2} \lambda_{3}+\frac{1}{2} & \frac{1}{2} \lambda_{3}+\frac{1}{2}
\end{array}\right] .
\end{gathered}
$$

Now consider the $(4,5)$ entry of $\mathbf{L}$, namely $-\frac{1}{2} \lambda_{3}+\frac{1}{2}$. The only possible cases are $\lambda_{3}=1$ or $\lambda_{3}=3$. In the former case we have a repeated eigenvalue, which is a contradiction, and therefore $\lambda_{3}=3$. The updated Laplacian is

$$
\mathbf{L}=\left[\begin{array}{ccccc}
\frac{1}{2} \lambda_{2}+\frac{1}{2} & -\frac{1}{2} \lambda_{2}+\frac{1}{2} & -1 & 0 & 0 \\
-\frac{1}{2} \lambda_{2}+\frac{1}{2} & \frac{1}{2} \lambda_{2}+\frac{1}{2} & -1 & 0 & 0 \\
-1 & -1 & 4 & -1 & -1 \\
0 & 0 & -1 & 2 & -1 \\
0 & 0 & -1 & -1 & 2
\end{array}\right]
$$

Finally, consider the $(1,2)$ entry of $\mathbf{L}$, namely $-\frac{1}{2} \lambda_{2}+\frac{1}{2}$. The only two possible cases are $\lambda_{2}=1$ or $\lambda_{2}=3$. In the former case, we have the repeated eigenvalue $\lambda_{2}=\lambda_{4}=1$ and in the latter case we have the repeated eigenvalue $\lambda_{2}=\lambda_{3}=3$. In either case, we obtain a contradiction. This completes the proof of Case 1.

Case 2. Suppose that the number of distinct indices where $\mathbf{b}_{1}, \mathbf{b}_{2}, \mathbf{b}_{3}$ are nonzero is 4 , say at $(i, j),(k, \ell),(i, k)$, respectively. Then from $\mathbf{u}_{2} \perp\left\{\mathbf{b}_{1}, \mathbf{b}_{2}, \mathbf{b}_{3}\right\}$ we obtain the linear system

$$
\left[\begin{array}{llll}
1 & 1 & 0 & 0 \\
0 & 0 & 1 & 1 \\
1 & 0 & 1 & 0
\end{array}\right]\left[\begin{array}{l}
\mathbf{u}_{2}(i) \\
\mathbf{u}_{2}(j) \\
\mathbf{u}_{2}(k) \\
\mathbf{u}_{2}(\ell)
\end{array}\right]=\left[\begin{array}{l}
0 \\
0 \\
0 \\
0
\end{array}\right],
$$

whose solution up to a scalar is $\mathbf{u}_{2}(i)=\mathbf{u}_{2}(\ell)=1$ and $\mathbf{u}_{2}(j)=\mathbf{u}_{2}(k)=-1$. Since $\mathbf{u}_{2} \perp \mathbf{u}_{1}$, then after a possible permutation of the indices, we have that $\mathbf{u}_{2}=\left[\begin{array}{lllll}1 & 1 & -1 & -1 & 0\end{array}\right]^{T}$. Now, $\mathbf{u}_{2}$ is orthogonal to $\mathbf{b}_{1}=\left[\begin{array}{lllll}1 & 0 & 1 & 0 & 0\end{array}\right]^{T}, \mathbf{b}_{2}=$ $\left[\begin{array}{lllll}1 & 0 & 0 & 1 & 0\end{array}\right]^{T}, \mathbf{b}_{3}=\left[\begin{array}{lllll}0 & 1 & 1 & 0 & 0\end{array}\right]^{T}$, and $\mathbf{b}_{4}=\left[\begin{array}{lllll}0 & 1 & 0 & 1\end{array}\right]^{T}$, and this leaves six vectors $\left\{\mathbf{b}_{5}, \ldots, \mathbf{b}_{10}\right\}$ in $S_{2}$ that are annihilated by $\left\{\mathbf{u}_{3}, \mathbf{u}_{4}, \mathbf{u}_{5}\right\}$. Now, we may assume that $\mathbf{u}_{3} \perp \mathbf{e}_{3}$, and since $\mathbf{u}_{3} \perp\left\{\mathbf{u}_{1}, \mathbf{u}_{2}\right\}$, it is straight forward to show that $\mathbf{u}_{3}$ takes the form $\mathbf{u}_{3}=[a, b, 0, a+b,-2(a+b)]^{T}$, where $a, b \neq 0$.
Now, by the pigeon hole principle, $\mathbf{u}_{3}$ is orthogonal to at least two vectors in $\left\{\mathbf{b}_{5}, \ldots, \mathbf{b}_{10}\right\}$. Similar arguments as in Case 1. show that in fact $\mathbf{u}_{3}$ is orthogonal to four vectors in $\left\{\mathbf{b}_{5}, \ldots, \mathbf{b}_{10}\right\}$ and that $\mathbf{u}_{3}=\left[\begin{array}{lllll}-1 & 1 & 0 & 0 & 0\end{array}\right]^{T}$. Now, we may assume that $\mathbf{u}_{4} \perp \mathbf{e}_{1}$, and since also $\mathbf{u}_{4} \perp\left\{\mathbf{u}_{1}, \mathbf{u}_{2}, \mathbf{u}_{3}\right\}$, this implies that $\mathbf{u}_{4}=\left[\begin{array}{lllll}0 & 0 & -1 & 1 & 0\end{array}\right]^{T}$. This then fixes $\mathbf{u}_{5}=[-1,-1,-1,-1,4]^{T}$. The proof that $\mathbf{L}$ has a repeated eigenvalue is similar as in Case 1.

Case 3. Suppose that the number of distinct indices where $\mathbf{b}_{1}, \mathbf{b}_{2}, \mathbf{b}_{3}$ are nonzero is 5 , say at $(i, j),(k, \ell),(m, k)$, respectively. Then from $\mathbf{u}_{2} \perp\left\{\mathbf{b}_{1}, \mathbf{b}_{2}, \mathbf{b}_{3}\right\}$ we obtain the linear system

$$
\left[\begin{array}{lllll}
1 & 1 & 0 & 0 & 0 \\
0 & 0 & 1 & 1 & 0 \\
0 & 0 & 1 & 0 & 1
\end{array}\right]\left[\begin{array}{c}
\mathbf{u}_{2}(i) \\
\mathbf{u}_{2}(j) \\
\mathbf{u}_{2}(k) \\
\mathbf{u}_{2}(\ell) \\
\mathbf{u}_{2}(m)
\end{array}\right]=\left[\begin{array}{l}
0 \\
0 \\
0 \\
0 \\
0
\end{array}\right]
$$

whose solution space is 2-dimensional and spanned by the vectors $\mathbf{v}_{1}=\left[\begin{array}{lllll}-1 & 1 & 0 & 0 & 0\end{array}\right]^{T}$ and $\mathbf{v}_{2}=\left[\begin{array}{lllll}0 & 0 & -1 & 1 & 1\end{array}\right]^{T}$. Since the entries of $\mathbf{u}_{2}$ sum up to one, we have that $\mathbf{u}_{2}=$ $\left[\begin{array}{lllll}-1 & 1 & 0 & 0 & 0\end{array}\right]^{T}$. The rest of the proof is then identical as in Case 1. This completes the proof of $n=5$.

\section{Proof of Proposition 4.3}

The proof is by induction on $r \in \mathbb{N}$. Let $i, j \in \mathcal{V}$ and suppose that $1 \leq \mathrm{d}_{\mathcal{G}}(i, j)$. Then necessarily $i \neq j$ and thus $\left(\mathbf{L}^{0}\right)_{i j}=0$ and $(\mathbf{L})_{i j}=(\mathbf{D})_{i j}-(\mathbf{A})_{i j}=-(\mathbf{A})_{i j}$. This proves the claim for $r=1$. Suppose by induction that the claim holds for $r \geq 1$. Fix vertices $i, j$ with $r+1 \leq \mathrm{d}_{\mathcal{G}}(i, j)$. Clearly, $r \leq$ $\mathrm{d}_{\mathcal{G}}(i, \bar{j})$ and therefore, by the induction hypothesis, $\left(\mathbf{L}^{k}\right)_{i j}=\overline{0}$ if $0 \leq k<r$, and in particular, $\left(\mathbf{L}^{r-1}\right)_{i j}=0$. Let $\mathcal{N}_{i}$ denote the neighbors of $i$. Then, for any $k \geq 1$ we have

$$
\begin{aligned}
\left(\mathbf{L}^{k}\right)_{i j}=\mathbf{e}_{i}^{T} \mathbf{L}^{k} \mathbf{e}_{j} & =\mathbf{e}_{i}^{T} \mathbf{D} \mathbf{L}^{k-1} \mathbf{e}_{j}-\mathbf{e}_{i}^{T} \mathbf{A} \mathbf{L}^{k-1} \mathbf{e}_{j}, \\
& =d_{i} \mathbf{e}_{i}^{T}\left(\mathbf{L}^{k-1}\right) \mathbf{e}_{j}-\sum_{\ell \in \mathcal{N}_{i}} \mathbf{e}_{\ell}^{T} \mathbf{L}^{k-1} \mathbf{e}_{j}, \\
& =d_{i}\left(\mathbf{L}^{k-1}\right)_{i j}-\sum_{\ell \in \mathcal{N}_{i}}\left(\mathbf{L}^{k-1}\right)_{\ell j} .
\end{aligned}
$$

Now, for each $\ell \in \mathcal{N}_{i}$ it is clear that $r \leq \mathrm{d}_{\mathcal{G}}(\ell, j)$. Hence, we can apply the induction hypothesis for each $\ell \in \mathcal{N}_{i}$, and in particular, $\left(\mathbf{L}^{r-1}\right)_{\ell j}=0$ and $\left(\mathbf{L}^{r}\right)_{\ell j}=(-1)^{r}\left(\mathbf{A}^{r}\right)_{\ell j}$. Therefore,

$$
\left(\mathbf{L}^{r}\right)_{i j}=d_{i}\left(\mathbf{L}^{r-1}\right)_{i j}-\sum_{\ell \in \mathcal{N}_{i}}\left(\mathbf{L}^{r-1}\right)_{\ell j}=0
$$

and consequently

$$
\left(\mathbf{L}^{r+1}\right)_{i j}=d_{i}\left(\mathbf{L}^{r}\right)_{i j}-\sum_{\ell \in \mathcal{N}_{i}}\left(\mathbf{L}^{r}\right)_{\ell j}=-\sum_{\ell \in \mathcal{N}_{i}}\left(\mathbf{L}^{r}\right)_{\ell j} .
$$

Hence

$$
\begin{aligned}
\left(\mathbf{L}^{r+1}\right)_{i j}=-\sum_{\ell \in \mathcal{N}_{i}}(-1)^{r}\left(\mathbf{A}^{r}\right)_{\ell j} & =(-1)^{r+1} \sum_{\ell \in \mathcal{N}_{i}} \mathbf{e}_{\ell}^{T} \mathbf{A}^{r} \mathbf{e}_{j}, \\
& =(-1)^{r+1}\left(\mathbf{e}_{i}^{T} \mathbf{A}\right) \mathbf{A}^{r} \mathbf{e}_{j}, \\
& =(-1)^{r+1} \mathbf{e}_{i}^{T}\left(\mathbf{A}^{r+1}\right) \mathbf{e}_{j}, \\
& =(-1)^{r+1}\left(\mathbf{A}^{r+1}\right)_{i j} .
\end{aligned}
$$

This ends the proof. 